\title{
gु \\ Thermoelectric effects in magnetic nanostructures
}

\author{
Moosa Hatami and Gerrit E. W. Bauer \\ Kavli Institute of NanoScience, Delft University of Technology, Lorentzweg 1, 2628 CJ Delft, The Netherlands \\ Qinfang Zhang and Paul J. Kelly \\ Faculty of Science and Technology and MESA+ Institute for Nanotechnology, University of Twente, P.O. Box 217, \\ 7500 AE Enschede, The Netherlands
}

(Received 1 January 2009; revised manuscript received 23 April 2009; published 20 May 2009)

\begin{abstract}
We model and evaluate the Peltier and Seebeck effects in magnetic multilayer nanostructures by a finiteelement theory of thermoelectric properties. We present analytical expressions for the thermopower and the current-induced temperature changes due to Peltier cooling/heating. The thermopower of a magnetic element is in general spin polarized, leading to spin-heat coupling effects. Thermoelectric effects in spin valves depend on the relative alignment of the magnetization directions and are sensitive to spin-flip scattering as well as inelastic collisions in the normal-metal spacer.
\end{abstract}

DOI: 10.1103/PhysRevB.79.174426

PACS number(s): 72.15.Jf, 75.30.Sg, 85.75.-d

\section{INTRODUCTION}

The Peltier effect refers to the conversion of an electric voltage into a temperature difference (that can be used for refrigeration), while the Seebeck effect refers to the inverse process, the generation of an electric field by a temperature gradient. ${ }^{1}$ Renewed interest in thermoelectric properties is motivated in part by the improved performance of nanometer-scale structures. ${ }^{2-5}$ Thin-film thermoelectric coolers can provide cheap and fast spot cooling in micro- and nanoelectronic circuits and devices. ${ }^{6,7}$ Strongly enhanced thermopower in quantum point contacts with widths approaching the Fermi wavelength can be used for sensitive and local electron thermometry. ${ }^{8,9}$ In ferromagnets and heterostructures involving magnetic elements, the effect of the magnetization (spin) degree of freedom on thermoelectric transport has to be taken into account. ${ }^{10-12}$ The giant magnetothermoelectric power in multilayered nanopillars, ${ }^{13,14}$ thermally excited spin currents in metals with embedded ferromagnetic clusters, ${ }^{15,16}$ and thermal spin-transfer torque in spin-valve devices ${ }^{17}$ are examples of spin-dependent thermoelectric phenomena on a nanometer scale.

Recently a large Peltier effect was discovered in transition-metal multilayered nanopillars by Fukushima et $a l .{ }^{18,19}$ The temperature and energy dissipation as a function of an applied current were monitored using the temperaturedependent electrical resistance. In asymmetric structures the parabolic dependence of the resistance arising from currentinduced Joule heating was found to be modified by a superimposed linear (Peltier) term that shifts the minimum resistance to a finite value of the current that could be positive or negative, depending on the combination of materials. Gravier et al. ${ }^{20}$ used a model of diffuse thermoelectric transport in (nonmagnetic) metallic heterostructures to compute the Peltier effect. These calculations had to be carried out numerically, and magnetism was not taken into account. The sample cross sections were used as fitting parameters that appeared to be too small compared to the actual sample sizes. ${ }^{18,19}$ This discrepancy was attributed to the neglect of interface scattering. Katayama-Yoshida et al. ${ }^{21}$ interpreted the perceived enhancement of the cooling power as a contribution from an adiabatic spin-entropy expansion term $\left(k_{B} / e\right) \ln 2$. Dubi and Di Ventra ${ }^{22}$ studied the Seebeck effect in single level quantum dots with ferromagnetic contacts.

Enhancing the performance of solid state cooling elements remains a challenge both for theory and experiment. ${ }^{1-7}$ Fukushima et al. ${ }^{18,19}$ suggested that the Peltier effect in transition-metal nanostructures could be useful for cooling magnetoelectronic devices. In order to assess this idea, the material dependence of the Peltier effect in magnetic nanostructures has to be understood. In this paper we investigate the Peltier effect in magnetic heterostructures theoretically, taking into account spin-dependent interface and bulk scattering by means of an extended finite-element (circuit) theory of transport ${ }^{17}$ that is a generalization of magnetoelectronic circuit theory. ${ }^{23-25}$ Such a theory is also suitable to study the magnetothermoelectric power in magnetic multilayers in which transport is normal to the interfaces. ${ }^{13,14}$

In the following we explain the method, initially disregarding bulk impurity scattering, which is justified in the thin layer limit when interface scattering is dominant. We start with a simple normal-metal $N_{1}|N| N_{2}$ trilayer structure in which metal $N$ is sandwiched between two electron reservoirs consisting of different metals $N_{1(2)}$. We then extend the methodology by making first one and then both reservoirs magnetic. In the $F_{1}|N| F_{2}$ (ferromagnet|normal-metall ferromagnet) spin valve sketched in Fig. 1, the relative orientation of the magnetization directions as well as the

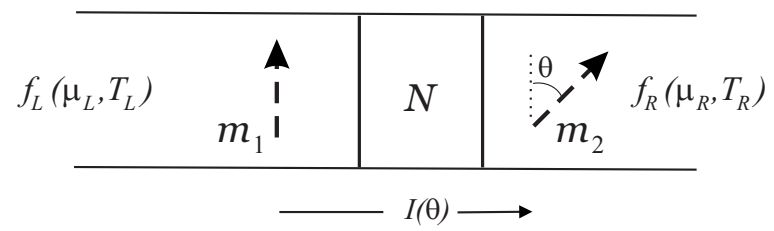

FIG. 1. Schematic of a spin-valve structure connected to reservoirs at different chemical potentials and/or temperatures $\left(f_{L(R)}\right.$ are the Fermi-Dirac distribution functions). Charge and heat flows through the normal-metal spacer $(N)$ are functions of the angle between the magnetization directions $(\theta)$ in the ferromagnets. 
strength of the inelastic collisions become important parameters. Finally, we show how bulk impurity scattering (normal as well as spin flip) can be introduced into the formalism, discuss the relevance of our results for experiments, and finish with a summary and conclusions.

\section{CURRENT-INDUCED ELECTRON COOLING AND HEATING}

A resistor network theory is an efficient way to describe the electric transport properties of magnetic heterostructures. Used to model the giant magnetoresistance effect in terms of the two-channel series resistor model, ${ }^{26,27}$ it has been generalized to include the spin-transfer torque in noncollinear magnetization configurations. ${ }^{25}$ A resistor model for thermoelectric effects was introduced by MacDonald ${ }^{28}$ to understand the effects of different types of impurities in homogeneous bulk metals.

In the present section we develop a generalized (spinless) circuit theory to describe the effects of temperature and voltage bias on normal-metal heterostructures. Our starting point is a (nonunique) definition of the circuit or device topology by partitioning it into reservoirs, resistors, and nodes. Discrete resistive elements are interfaces, potential barriers, or constrictions that limit the transport. We require that these resistors can be represented by purely elastic scattering processes. The resistors are separated by nodes in which electrons can be described by semiclassical distribution functions, $f_{i}$, in node $i$. When inelastic electron-electron or electron-phonon interactions in the nodes are strong enough, the $f_{i}$ become Fermi-Dirac distributions parametrized by temperatures $T_{i}$ and chemical potentials $\mu_{i}$. The charge and heat currents through a given resistor, denoted by $I$ $=\int d \epsilon l(\epsilon)$ and $e \dot{Q}=\int d \epsilon(\epsilon-\mu)_{l}(\epsilon)=e I^{E}-\mu I$, respectively, where $\mu$ is the global chemical potential in equilibrium, are determined via the spectral current density

$$
\iota(\epsilon)=G(\epsilon)\left[f_{L}(\epsilon)-f_{R}(\epsilon)\right],
$$

where $G(\epsilon)$ is an energy-dependent (spectral) conductance between two neighboring nodes with distribution functions $f_{L(R)}$. According to Landauer-Büttiker scattering theory the conductance,

$$
G(\epsilon)=\frac{2 e^{2}}{h} \sum_{m n}\left[\delta_{m n}-r_{m n}^{*}(\epsilon) r_{m n}(\epsilon)\right],
$$

depends on the energy-dependent reflection amplitudes $r_{m n}(\epsilon)$ at the resistor for electrons that are incident from a node or reservoir. $2 e^{2} / h \simeq 1 / 13 \mathrm{k} \Omega$ is the quantum of conductance. For structure elements such as interfaces which are not overly complicated, $r_{m n}(\epsilon)$ can be calculated from microscopic, first-principles calculations. ${ }^{17-30}$ Thermoelectric effects arise from the energy dependence of $G(\epsilon)$. The circuit theory approach requires that scattering in the resistive elements is elastic. $G(\epsilon)$ may in principle be bias dependent, which becomes important for tunnel junctions. Here we concentrate on abrupt intermetallic interfaces with biasindependent spectral conductances. The interface resistance of transparent interfaces in a diffuse environment is affected by a correction caused by the drift of the distribution function. ${ }^{25,31}$ The bare spectral resistance $G(\epsilon)^{-1}$ is then substituted by $G(\epsilon)^{-1}-\left[G_{N_{L}}(\epsilon)^{-1}+G_{N_{R}}(\epsilon)^{-1}\right]$, where $G_{N_{i}}(\epsilon)$ $=2 e^{2} M_{N_{i}}(\epsilon) / h$ are the Sharvin conductances of the metals that form the interface and $M_{N_{i}}$ are the total numbers of single-spin transport modes.

Let us now consider a nonequilibrium steady state in a simple $N_{1}|N| N_{2}$ normal-metal structure in which the chemical potential $\mu_{1(2)}=\mu-e V_{1(2)}$ and temperature $T_{1(2)}$ of the nodes deviate from their equilibrium values $(\mu, T)$. Node $N$ is assumed to be fully thermalized with a distribution described by $\left(e V_{N}, T_{N}\right)$ that still have to be determined. To lowest order in the applied thermoelectric fields, we use the expansions $f_{i} \approx f_{0}+\frac{\partial f_{0}}{\partial \mu}\left(\mu_{i}-\mu\right)+\frac{\partial f_{0}}{\partial T}\left(T_{i}-T\right)$ in the following, where $f_{0}$ is the equilibrium Fermi-Dirac distribution function. It is possible to proceed and compute thermoelectric properties for arbitrary energy dependences of $G(\epsilon)$. However, results become much simpler upon using the Sommerfeld expansion ${ }^{32}$ in $k_{B} T / \epsilon_{F}$. Provided the conductances do not vary too rapidly near the Fermi energy [to be precise $\left.\partial_{\epsilon} G\right|_{\epsilon_{F}} \neq \infty$ and $\left.\left.G(\epsilon) e^{-\epsilon / k_{B} T}\right|_{\epsilon \gg \epsilon_{F}} \rightarrow 0\right]$, the following approximation is applied:

$$
\begin{aligned}
& \int_{-\infty}^{\infty} d \xi\left(-\frac{\partial f_{0}}{\partial \xi}\right) \xi^{n} G(\xi) \\
& \approx G(0) \delta_{n, 0}+\frac{\pi^{2}}{6}\left(k_{B} T\right)^{2}\left[n(n-1) \xi^{n-2} G(\xi)\right. \\
&\left.+2 n \xi^{n-1} \partial_{\xi} G(\xi)+\xi^{n} \partial_{\xi}^{2} G(\xi)\right]_{\xi=0},
\end{aligned}
$$

where $\xi$ is the energy variable relative to the Fermi energy. Applying the Sommerfeld expansion to the expressions for the charge and heat currents in terms of the spectral current density [Eq. (1)] results in expressions for the charge and heat currents into the normal node through junctions 1 and 2 , valid for $\left.\left.\frac{\pi^{2}}{6}\left(k_{B} T\right)^{2} \partial_{\epsilon}^{2} G\right|_{\epsilon_{F}} \ll G\right|_{\epsilon_{F}}$ as follows:

$$
\left(\begin{array}{l}
I_{1(2)} \\
\dot{Q}_{1(2)}
\end{array}\right)=G_{1(2)}\left(\begin{array}{cc}
1 & S_{1(2)} \\
-S_{1(2)} T & -\mathcal{L}_{0} T
\end{array}\right)\left(\begin{array}{c}
V_{N}-V_{1(2)} \\
T_{N}-T_{1(2)}
\end{array}\right) \text {, }
$$

where $\mathcal{L}_{0}=\left(k_{B} / e\right)^{2} \pi^{2} / 3 \simeq 2.45 \times 10^{-8} \mathrm{~V}^{2} \mathrm{~K}^{-2}$ is the Lorenz number. $G_{i}=G_{i}\left(\epsilon_{F}\right)$ are the conductances and $S_{i}=$ $-\left.e \mathcal{L}_{0} T \partial_{\epsilon} \ln G_{i}\right|_{\epsilon_{F}}$ (Mott's formula) are the Seebeck coefficients or thermopowers at the zero-temperature chemical potential, which for metals is just the Fermi energy $\epsilon_{F}$. In bulk materials the thermopower can be positive or negative and even change sign as a function of the temperature. ${ }^{33}$

The chemical potential shift $e V_{N}$ and temperature $T_{N}$ of the central normal node and thus the thermal distribution function $f_{N}$ are determined by conservation laws: the charge and energy flows of the electrons are conserved, $I_{1}+I_{2}=0$ and $\dot{Q}_{1}+\dot{Q}_{2}=0$. The latter is affected in principle by the phonon heat conduction through the contacts (see Appendix A) but is disregarded here since thermal transport in good metals is dominated by the conduction electrons. ${ }^{34}$ Using Eq. (4) and the conservation laws we find to lowest order in the 
charge current $I$ that the electron temperature $T_{N}$ of the normal island is modified from the zero-charge current value $T_{0}$ as

$$
T_{N}=T_{0}+\frac{\left(\Pi_{1}-\Pi_{2}\right) I}{\kappa_{1}+\kappa_{2}} .
$$

The electron cooling or heating of asymmetric structures $\left(\Pi_{1} \neq \Pi_{2}\right)$ by the applied current is the Peltier effect. $\Pi_{1(2)}$ $=S_{1(2)} T$ are the Peltier coefficients and

$$
\kappa_{1(2)}=\mathcal{L}_{0} T G_{1(2)}\left(1-S_{1(2)}^{2} / \mathcal{L}_{0}\right)
$$

are the thermal conductances of the resistive elements. Large thermopowers violate the simple proportionality between electrical and heat conductance, $\kappa=\mathcal{L}_{0} T G$, the WiedemannFranz law. The expression for the zero-current temperature in the central node,

$$
T_{0}=\frac{\kappa_{1} T_{1}+\kappa_{2} T_{2}}{\kappa_{1}+\kappa_{2}},
$$

follows from energy conservation. The charge current $I$ $=I_{\Delta V}+I_{\Delta T}$ is excited by a voltage difference $\Delta V=V_{2}-V_{1}$ as well as the temperature bias $\Delta T=T_{2}-T_{1}$. The total electric charge and heat currents are then given by

$$
\begin{gathered}
I=\bar{G}(\Delta V+\bar{S} \Delta T), \\
\dot{Q}=-\bar{\Pi} I-\bar{\kappa} \Delta T,
\end{gathered}
$$

where the total conductance becomes

$$
\bar{G}=\frac{G_{1} G_{2}\left(\kappa_{1}+\kappa_{2}\right)}{G_{1} \kappa_{1}+\left(G_{1}+G_{2}\right) \kappa_{12}+G_{2} \kappa_{2}},
$$

with

$$
\kappa_{12} \equiv 2 \mathcal{L}_{0} T \frac{G_{1} G_{2}}{G_{1}+G_{2}}\left(1-\frac{S_{1} S_{2}}{\mathcal{L}_{0}}\right)
$$

The total conductance/resistance $(\bar{R}=1 / \bar{G})$ violates the series resistor rule, $\bar{R} \neq R_{1}+R_{2}$ (with $R_{i}=1 / G_{i}$ ), which is only recovered when either $S_{1}=S_{2}$ or when $S_{i}^{2} \ll \mathcal{L}_{0}$; the latter is always the case at low temperatures. On the other hand, the total thermopower $\bar{S}$ (or Peltier coefficient $\bar{\Pi}=\bar{S} T$ ) and thermal conductance $\bar{\kappa}$ do obey simple sum rules,

$$
\begin{aligned}
& \frac{\bar{S}}{\bar{\kappa}}=\frac{S_{1}}{\kappa_{1}}+\frac{S_{2}}{\kappa_{2}}, \\
& \frac{1}{\bar{\kappa}}=\frac{1}{\kappa_{1}}+\frac{1}{\kappa_{2}} .
\end{aligned}
$$

When in series, two thermal or electrical resistances are additive, as expressed in Eq. (13) or $\bar{R}=R_{1}+R_{2}$ which is valid in the limit $S_{i}^{2} \ll \mathcal{L}_{0}$. In contrast, the thermopower [Eq. (12)] depends on the spatial distribution of the scattering objects rather than its integral (the thermopower in a homogeneous bulk metal does not depend on its length). Equation (12) holds not only for the spatially distributed scatterers consid- ered here but can also describe the relative contributions of different types of scatterers to the thermopower in bulk materials. ${ }^{28}$

The second law of thermodynamics (a non-negative entropy production) requires $\kappa>0$, which in the Sommerfeld approximation leads to ${ }^{35}\left|S_{\text {max }}\right|=\sqrt{\mathcal{L}_{0}} \simeq 157 \mu \mathrm{V} / \mathrm{K}$. Defining the thermoelectric figure of merit, $Z T=\left|\bar{S} \Delta T_{N} / \Delta V\right|$, [Eq. (5)] for the temperature change $\Delta T_{N}=T_{N}-T_{0}$ induced by an applied voltage results in

$$
Z T=\frac{1}{4}\left|\frac{\bar{S}\left(S_{1}-S_{2}\right)}{\mathcal{L}_{0}-\bar{S}^{2}}\right|,
$$

assuming $G_{1}=G_{2}$ and $\left(S_{1}-S_{2}\right) S_{1(2)} \ll \mathcal{L}_{0}$ so that $\left|\bar{S}_{\text {max }}\right|=\sqrt{\mathcal{L}_{0}}$ corresponds to the maximum efficiency $Z T=\infty$. Even for the best thin-film thermoelectric materials ${ }^{3} Z T \lesssim 3$, and for most metallic structures $S^{2} \ll \mathcal{L}_{0}$. Quantum point contacts, however, have larger thermopowers due to size quantization, ${ }^{8,9}$ so $S^{2}$ can be comparable to $\mathcal{L}_{0}$ and the predicted effects should be observable in nanoscale structures.

Equation (5) holds for low current densities for which $\delta V / 2 \ll \Pi \ll 2 \mathcal{L}_{0} T^{2} / \delta V$, where $\delta V$ is the voltage drop over a single contact. Nonlinear heating by applied currents can be included my means of the quadratic term in the expansions of the distribution functions, i.e., $f_{i} \approx f_{0}+\frac{\partial f_{0}}{\partial \mu}\left(\mu_{i}-\mu\right)+\frac{\partial f_{0}}{\partial T}\left(T_{i}\right.$ $-T)+\frac{1}{2} \frac{\partial^{2} f_{0}}{\partial \mu^{2}}\left(\mu_{i}-\mu\right)^{2}+\cdots$, and using the approximation $\int_{-\infty}^{\infty} d \xi\left(\partial_{\xi}^{2} f_{0}\right) \xi G(\xi) \approx G(0)$. When $\Pi \ll \delta V \ll \mathcal{L}_{0} T^{2} / \Pi$, expressions for the nonlinear currents reduce to

$$
\left(\begin{array}{c}
I_{1(2)} \\
\dot{Q}_{1(2)}
\end{array}\right) \simeq G_{1(2)}\left(\begin{array}{cc}
1 & S_{1(2)} \\
-\Pi_{1(2)}-\widetilde{V}_{1(2)} & -\mathcal{L}_{0} \widetilde{T}_{1(2)}
\end{array}\right)\left(\begin{array}{c}
V_{N}-V_{1(2)} \\
T_{N}-T_{1(2)}
\end{array}\right),
$$

where $\widetilde{V}_{1(2)}=\left(V_{N}+V_{1(2)}\right) / 2$ and $\widetilde{T}_{1(2)}=\left(T_{N}+T_{1(2)}\right) / 2$. For reservoir temperatures $T_{1}=T_{2}=T$ and in the absence of the thermopowers $S_{1(2)}=0$, or when Joule heating dominates, particle and energy current conservation requires $\left(G_{1}+G_{2}\right) V_{N}$ $=G_{1} V_{1}+G_{2} V_{2}$ and

$$
T_{N}^{2}=T^{2}+\frac{(\Delta V)^{2}}{\mathcal{L}_{0}} \frac{G_{1} G_{2}}{\left(G_{1}+G_{2}\right)^{2}}
$$

so that we recover the result for the maximum amplitude of the electron temperature profile in the middle of a diffusive bulk wire (with the conductance $G_{1}=G_{2}=G$ ) due to heating by inelastic electron-electron collisions. ${ }^{36,37}$ By taking into account the thermopowers of the junctions, but in the limit $S^{2} \ll \mathcal{L}_{0}$, the change in the electron temperature in the island is found as

$$
\begin{aligned}
\Delta T_{N}= & \frac{\left(\Pi_{1}-\Pi_{2}\right) I}{\mathcal{L}_{0} T\left(G_{1}+G_{2}\right)}+\frac{\bar{R} I^{2}}{2 \mathcal{L}_{0} T\left(G_{1}+G_{2}\right)} \\
& +\mathcal{O}\left(\left(\frac{\Delta T}{T}\right)^{2}, \frac{\Delta T}{T} \frac{(\Delta V)^{2}}{\mathcal{L}_{0} T^{2}}, \frac{S(\Delta V)^{3}}{\mathcal{L}_{0}^{2} T^{3}}, \cdots\right)
\end{aligned}
$$

in which the electric current [Eq. (8)] is driven by a voltage and/or temperature bias. Equations (16) and (17) yield the 
same result for a small temperature increase due to Joule heating when $\Pi_{1}=\Pi_{2}$ and $T_{1}=T_{2}$.

Following Fukushima et al. ${ }^{18,19}$ we use Eq. (17) to derive an expression for the Peltier coefficient in terms of a critical current $I_{p}$ at which heating and cooling cancel each other. Assuming that the resistance scales linearly with the electron temperature in the node, $\Delta T_{N}=0$ leads to

$$
\left(\Pi_{2}-\Pi_{1}\right) I_{p}=\frac{\bar{R} I_{p}^{2}}{2} .
$$

The factor $1 / 2$ on the right-hand side implies that the current heating is only half as large as considered by Refs. 18 and 19 , whereas the "cooling power" $\bar{R} I_{p}=2\left(\Pi_{2}-\Pi_{1}\right)$ (in units of $\mathrm{mV}$ ) is twice as large. This discrepancy can be explained as follows. In our model, the energy is dissipated in the nodes and reservoirs of the device, not at the sharp interfaces. We monitor the temperature change in the normal-metal node which is assumed to be effectively thermalized. Half of the generated heat is dissipated in the reservoirs that by definition do not contribute to the resistance change. The expressions of Fukushima et al. ${ }^{18,19}$ can be recovered by treating the highly resistive junctions in their samples as bulk material in which heat is generated and contributes to its temperature and resistance rise (see Sec. VII).

\section{PELTIER AND SEEBECK EFFECTS IN THE PRESENCE OF A SINGLE FERROMAGNETIC ELEMENT}

The thermoelectric transport [Eq. (4)] can be generalized to include the spin degree of freedom. For spin-dependent thermoelectric transport through an $F \mid N$ interface the spinpolarized electric charge and heat currents read as

$$
\left(\begin{array}{c}
I^{\uparrow(\downarrow)} \\
\dot{Q}^{\uparrow(\downarrow)}
\end{array}\right)=G^{\uparrow(\downarrow)}\left(\begin{array}{cc}
1 & S^{\uparrow(\downarrow)} \\
-S^{\uparrow(\downarrow)} T & -\mathcal{L}_{0} T
\end{array}\right)\left(\begin{array}{c}
V_{N}^{\uparrow(\downarrow)}-V_{F}^{\uparrow(\downarrow)} \\
T_{N}^{\uparrow(\downarrow)}-T_{F}^{\uparrow(\downarrow)}
\end{array}\right),
$$

where the spin dependence of the conductance $G^{\uparrow(\downarrow)}$, thermopower $S^{\uparrow(\downarrow)}=-\left.e \mathcal{L}_{0} T \partial_{\epsilon} \ln G^{\uparrow(\downarrow)}\right|_{\epsilon_{F}}$, heat current, and temperature is expressed by the superscript $\uparrow(\downarrow)$ for majority (minority) spin electrons. $V_{s}=V^{\uparrow}-V^{\downarrow}$ is the particle spin accumulation. Referring to the discussion below we conjecture the existence of a heat spin accumulation $T_{s}=T^{\uparrow}-T^{\downarrow}$, i.e., a temperature imbalance for majority and minority electrons, when thermalization is weak. We also define the total thermopower $S=-(\Delta V / \Delta T)_{I=0}$ of an interface between a normal metal and a ferromagnet as

$$
S=-e \mathcal{L}_{0} T\left(\frac{\partial_{\epsilon} G}{G}\right)_{\epsilon_{F}}=\left(\frac{G^{\uparrow} S^{\uparrow}+G^{\downarrow} S^{\downarrow}}{G^{\uparrow}+G^{\downarrow}}\right)_{\epsilon_{F}} .
$$

This thermopower is observable when the interface is part of a (hetero) Sharvin point contact in direct contact with large reservoirs that prevent buildup of a spin accumulation. In a diffusive environment, however, the local spin accumulation should be taken into account, as described in the following. The spin polarization of the interface thermopower is defined as

$$
P_{S} \equiv \frac{S^{\uparrow}-S^{\downarrow}}{S^{\uparrow}+S^{\downarrow}}=\frac{P^{\prime}-P}{1-P^{\prime} P},
$$

where $P$ and $P^{\prime}$ are the polarizations of the conductance $G^{\uparrow(\downarrow)}$ and its energy derivative $\partial_{\epsilon} G^{\uparrow(\downarrow)}$, respectively-both at the Fermi energy. Whereas $|P|<1$ and $\left|P^{\prime}\right| \gg 1$ when $\partial_{\epsilon} G^{\uparrow}$ approaches $-\partial_{\epsilon} G^{\downarrow}$. $P_{S}$ is also in principle unbounded. Using

$$
-e \mathcal{L}_{0} T\left(\frac{\partial P}{\partial \epsilon}\right)_{\epsilon_{F}}=S\left(P^{\prime}-P\right)_{\epsilon_{F}},
$$

it follows that $P_{S} \neq 0$ when the conductance polarization is energy dependent. Spin polarization of the thermopower of ferromagnetic materials ${ }^{38-40}$ has been invoked to, e.g., explain the giant magnetothermoelectric effect of magnetic multilayers. ${ }^{41}$ For a few combinations of materials, the interface thermopower and its spin polarization are known from first-principles calculations. ${ }^{17,30}$

Consider now an $F_{1}|N| N_{2}$ pillar with one magnetic contact. Conservation of charge, spin, and energy currents implies the Kirchhoff rules $I_{1 \alpha}+I_{2 \alpha}=0$ and $\Sigma_{\alpha} \dot{Q}_{1}^{\alpha}+\dot{Q}_{2}^{\alpha}=0$, where $\alpha=\uparrow(\downarrow)$. The individual spin currents are separately conserved since we disregard spin-flip scattering in the normal-metal spacer when the length of the metal does not exceed its spin-diffusion length. In contrast, the heat spin accumulation, i.e., the temperature difference between the two spin species on the central island, is assumed to vanish by strong inelastic scattering, which is likely for temperatures that are not too low and/or metals that are not too clean. In this regime the electron temperature on the island becomes

$$
T_{N}=T_{0}+\frac{\left(\gamma_{1} S_{1}-S_{2}\right) I}{\mathcal{L}_{0}\left(G_{1}+G_{2}\right)}
$$

We may call

$$
\gamma_{1}=\frac{\left(1-P^{\prime} P\right)+G_{2} / G_{1}}{\left(1-P^{2}\right)+G_{2} / G_{1}}
$$

a "spin-entropy factor," because it reflects the spin polarization of the entropy flow per unit of the electric current (the thermopower), $S^{\uparrow} \neq S^{\downarrow}\left(P^{\prime} \neq P\right)$. In the limit $S_{\uparrow(\downarrow)}^{2} \ll L_{0}$ (and therefore $\left.\kappa^{\uparrow(\downarrow)} \approx L_{0} T G^{\uparrow(\downarrow)}\right)$ the temperature $T_{0}$ [Eq. (7)] is not affected by the magnetism. When $P^{\prime} \neq P$ the Peltier cooling (heating) does not vanish even when $S_{1}=S_{2}$. The thermopower spin polarization, $P_{S}$, can enhance or suppress the Peltier effect depending on the spin polarization $P$ and the relative amplitude of the conductances $G_{2} / G_{1} \cdot \gamma_{1}$ can become large when $\left|P^{\prime}\right| \gg 1$ (an example is $P^{\prime}$ at a disordered $\mathrm{Cr} \mid \mathrm{Fe}$ interface; see Table I).

The total thermopower can be expressed in terms of the properties of its constituent elements, in the limit $S^{\uparrow(\downarrow)} \ll \sqrt{\mathcal{L}_{0}}$ and for strongly thermalized electrons, as

$$
\frac{\bar{S}}{\bar{G}}=\frac{\gamma_{1} S_{1}}{G_{1}}+\frac{S_{2}}{G_{2}} \text {. }
$$

Therefore, when a spin accumulation is excited in the proximate normal metal, the magnetic junction contributes to the thermopower not by the Seebeck coefficient of the point 
TABLE I. Thermoelectric interface parameters calculated at the Fermi energy for a number of almost lattice-matched interfaces including Schep's drift correction (Ref. 31). With the exception of the $\mathrm{Cr} \mid \mathrm{Co}$ interface for which $\mathrm{Co}$ is assumed to be bcc, $\mathrm{Cr}$ and $\mathrm{Fe}$ are bcc while $\mathrm{Cu}, \mathrm{Co}$, and $\mathrm{Au}$ are assumed to be fcc. The asterisk $(*)$ indicates a dirty interface modeled in a $10 \times 10$ lateral supercell with two layers of $50 \%-$ $50 \%$ alloy.

\begin{tabular}{lcccccccrrr}
\hline \hline & $\left.\begin{array}{c}G \\
\left(10^{15}\right.\end{array} \Omega^{-1} \mathrm{~m}^{-2}\right)$ & $\begin{array}{c}S / T \\
\left(\mathrm{nV} / \mathrm{K}^{2}\right)\end{array}$ & $\begin{array}{c}P \\
(\%)\end{array}$ & $\begin{array}{c}P^{\prime} \\
(\%)\end{array}$ & $\begin{array}{c}P_{S} \\
(\%)\end{array}$ & $\eta_{R}$ & $\eta_{I}$ & $\eta_{R}^{\prime}$ & $\eta_{I}^{\prime}$ \\
\hline $\mathrm{Cu} \mid \mathrm{Co}(001)$ & 4.43 & -13 & 75 & 72 & -8 & 0.50 & -0.036 & 0.03 & 0.00 \\
$\mathrm{Cu} \mid \mathrm{Co}(001)^{*}$ & 4.29 & -34 & 74 & 89 & 43 & 0.49 & -0.054 & 0.06 & -0.01 \\
$\mathrm{Cu} \mid \mathrm{Co}(110)$ & 3.42 & -10 & 69 & 6 & -66 & 0.67 & -0.082 & -0.32 & 0.44 \\
$\mathrm{Cu} \mid \mathrm{Co}(110)^{*}$ & 3.52 & -13 & 64 & 85 & 45 & 0.63 & -0.077 & 0.07 & -0.05 \\
$\mathrm{Cu} \mid \mathrm{Co}(111)$ & 3.69 & -15 & 60 & 56 & -6 & 0.53 & -0.006 & 0.13 & 0.48 \\
$\mathrm{Cu} \mid \mathrm{Co}(111)^{*}$ & 3.42 & -15 & 68 & 77 & 17 & 0.64 & -0.073 & 0.13 & -0.05 \\
$\mathrm{Cr} \mid \mathrm{Au}(001)$ & 0.36 & 7 & 0 & 0 & 0 & & & & \\
$\mathrm{Cr} \mid \mathrm{Au}(001)^{*}$ & 0.67 & 0 & 0 & 0 & 0 & & & & \\
$\mathrm{Cr} \mid \mathrm{Fe}(001)$ & 0.88 & 22 & -74 & -40 & 48 & 4.23 & 1.38 & -4.27 & -1.38 \\
$\mathrm{Cr} \mid \mathrm{Fe}(001)^{*}$ & 0.94 & 7 & -53 & -190 & -9500 & 3.25 & 0.43 & -0.48 & 9.39 \\
$\mathrm{Cr} \mid \mathrm{Co}(001)$ & 0.56 & 62 & -62 & -111 & -160 & 3.03 & -0.59 & -2.86 & -3.46 \\
$\mathrm{Cr} \mid \mathrm{Co}(001)^{*}$ & 0.71 & 23 & -23 & -95 & -92 & 2.92 & -1.79 & -0.86 & 3.21 \\
\hline \hline
\end{tabular}

contact $S_{1}$ but by the product with the spin-entropy factor $\gamma_{1} S_{1}$.

\section{MAGNETO-PELTIER AND MAGNETOTHERMOPOWER IN SPIN VALVES}

We proceed to the study of thermoelectric effects in asymmetric $F_{1}\left(\mathbf{m}_{1}\right)|N| F_{2}\left(\mathbf{m}_{2}\right)$ spin valves (see Fig. 1) for arbitrary relative orientations of the magnetizations, $\mathbf{m}_{1} \cdot \mathbf{m}_{2}=\cos \theta$. The electron distributions in the nodes and reservoirs are now $2 \times 2$ matrices in spin space that can be expanded into scalar and vector components $\hat{f}^{F(N)}=f_{c}^{F(N)} \hat{1}+\hat{\boldsymbol{\sigma}} \cdot \mathbf{s}^{F(N)} f_{s}^{F(N)}$, where $\hat{\boldsymbol{\sigma}}$ is the vector of Pauli matrices and $\hat{1}$ is the $2 \times 2$ unit matrix. The unit vector of the spin quantization axis $\mathbf{s}^{F}$ is parallel to the magnetization of the ferromagnet, whereas $\mathbf{s}^{N}$ can point to any direction. In linear response, the $2 \times 2$ spectral current in spin space across a ferromagnet-normal-metal junction at energy $\epsilon$ in the absence of spin-flip and inelastic interface scattering is given as a spectral Landauer-Büttikertype expression, ${ }^{23,24}$

$$
\hat{\imath}_{N \mid F}(\boldsymbol{\epsilon})=\sum_{\alpha \beta} G^{\alpha \beta}(\epsilon) \hat{u}^{\alpha}\left[\hat{f}^{F}(\boldsymbol{\epsilon})-\hat{f}^{N}(\boldsymbol{\epsilon})\right] \hat{u}^{\beta},
$$

where $\hat{u}^{\uparrow(\downarrow)}=(\hat{1} \pm \hat{\boldsymbol{\sigma}} \cdot \mathbf{m}) / 2$ are projection matrices in which the unit vector $\mathbf{m} \equiv \mathbf{s}^{F}$ denotes the magnetization direction of the ferromagnet. The conductance tensor elements read as $G^{\alpha \beta}(\epsilon)=\left(e^{2} / h\right) \sum_{n m}\left[\delta_{m n}-r_{n m}^{\alpha}(\epsilon)\left(r_{n m}^{\beta}(\epsilon)\right)^{*}\right]$ in terms of the energy-dependent reflection coefficients $r_{n m}^{\alpha}(\epsilon)$ for majority and minority spins at the $N \mid F$ interface. Its diagonal elements are the conventional spin-dependent conductances that govern, e.g., the giant magnetoresistance, whereas the complex nondiagonal elements, the so-called spin-mixing conductances, parametrize the transverse spin currents that are absorbed by the ferromagnet and give rise to torques on the magnetization. The total charge-spin and heat matrix currents are defined as $\hat{I}=\int d \epsilon \hat{l}(\epsilon)$ and $e \hat{\dot{Q}}=\int d \epsilon(\epsilon-\mu) \hat{\imath}(\epsilon)=e \hat{I}^{\epsilon}-\mu \hat{I}$, respectively, where $\mu$ is the equilibrium chemical potential and $\hat{I}^{\epsilon}$ the energy current. In the following we assume that both spin components $f^{\uparrow(\downarrow)}=f_{c} \pm f_{s}$ of the diagonalized matrix distribution functions $\hat{f}^{F(N)}$ may be described by thermalequilibrium Fermi-Dirac distribution functions with spindependent chemical potentials and temperatures. The Sommerfeld expansion can then be employed to derive expressions for the transport currents as a function of applied voltage or temperature gradients in terms of the conductance tensor $G^{\alpha \beta}$ and its energy derivative $G_{\epsilon}^{\alpha \beta}$ at the Fermi energy. ${ }^{17}$ The total charge, spin, and heat currents read as $I_{c}=\operatorname{Tr}[\hat{I}], \mathbf{I}_{s}=\operatorname{Tr}[\boldsymbol{\sigma} \hat{I}]$, and $\dot{Q}_{c}=\operatorname{Tr}[\hat{\dot{Q}}]$, respectively, where the trace is over spin indices.

The charge and energy conservation laws read as $I_{c 1}$ $+I_{c 2}=0$ and $\dot{Q}_{c 1}+\dot{Q}_{c 2}=0$. Moreover, in the absence of spinflip scattering in the normal node, the total spin angular momentum current is conserved as well, i.e., $\mathbf{I}_{s 1}+\mathbf{I}_{s 2}=0$. These Kirchhoff Laws close the system of transport equations in the strongly thermalized regime. In what we call the weakly thermalized regime, the distributions for each spin species are thermalized separately, but the energy exchange between the spin subsystems is disregarded, which is a realistic scenario at low temperatures. ${ }^{42}$ In this limit a spin temperature vector on the central island exists, $\mathbf{T}_{s} \neq 0$, and we require $\dot{\mathbf{Q}}_{s 1}+\dot{\mathbf{Q}}_{s 2} \approx 0$ where $\dot{\mathbf{Q}}_{s}=\operatorname{Tr}[\boldsymbol{\sigma} \hat{\dot{Q}}]$, which means that energy is conserved for each spin channel separately. It is worthwhile to compare the thermoelectric transport properties such as the total conductance and thermopower of the spin-valve structure in the different interacting regimes.

In the strongly thermalized regime for a symmetric spin valve, $G_{1}^{\alpha \beta}=G_{2}^{\alpha \beta}$ and $G_{\varepsilon 1}^{\alpha \beta}=G_{\varepsilon 2}^{\alpha \beta}$, the temperature of the normal-metal island $T_{N}=T_{0}$ is not affected by electric current. The total electric current reads as ${ }^{17}$ 


$$
I_{c}=\frac{G}{2}(\Delta V+S \Delta T)-\frac{P G}{2} \frac{\tan ^{2} \theta / 2}{\eta_{R}+\tan ^{2} \theta / 2}\left(P \Delta V+P^{\prime} S \Delta T\right) .
$$

Here $\eta_{R}=2 \operatorname{Re} G^{\uparrow \downarrow} / G>0$, where $G^{\uparrow \downarrow}$ is the complex spinmixing conductance. ${ }^{25}$ For most metallic contacts $\eta_{I}$ $=2 \operatorname{Im} G^{\uparrow \downarrow} / G$ is small $\left(\eta_{I} \ll \eta_{R}\right)$ (Ref. 43) and is disregarded in the analytical results. However, since $\eta_{I}$ is not small for $\mathrm{Cr} \mid \mathrm{Fe}$ and $\mathrm{Cr} \mid \mathrm{Co}$ interfaces, see Table I, it is included in the numerical results for these junctions. The angular magnetoresistance for $\Delta T=0$ as measured by Urazhdin et al. ${ }^{44}$ is well described by circuit theory. ${ }^{45}$ The thermoelectric transport properties of the spin-valve structure differ significantly in the different interacting regimes. In the Sommerfeld approximation, the spin-mixing thermopower $S^{\uparrow \downarrow} \equiv-e \mathcal{L}_{0} T G_{\varepsilon}^{\uparrow \downarrow} / G^{\uparrow \downarrow}$ and the dimensionless mixing parameter $\eta_{R(I)}^{\prime}$ $\equiv 2 \operatorname{Re}(\operatorname{Im}) G_{\epsilon}^{\uparrow \downarrow} / G_{\epsilon}$ enter expressions for the electric currents only when $\mathbf{T}_{s}^{N} \neq 0$, i.e., in the weakly thermalized regime. ${ }^{17}$

In the presence of a temperature bias $\Delta T$ with an open electric circuit $\left(I_{c}=0\right)$, the induced thermoelectric voltage $\Delta V$ is described by the angular magnetothermopower (MTP) $\bar{S}(\theta)=-(\Delta V / \Delta T)_{I_{c}=0}$ which in the strongly thermalized regime reads as ${ }^{17}$

$$
\begin{gathered}
\frac{\bar{S}(\theta)}{S}=\frac{\eta_{R}+\left(1-P P^{\prime}\right) \tan ^{2} \theta / 2}{\eta_{R}+\left(1-P^{2}\right) \tan ^{2} \theta / 2}, \\
\mathrm{MTP} \equiv \frac{\bar{S}(\pi)-\bar{S}(0)}{S}=\frac{-P P_{S}}{1+P P_{S}} .
\end{gathered}
$$

The MTP is finite when the interface thermopower is spin polarized, $P_{S} \neq 0$. When $P P^{\prime}>1$ (which also requires $P_{S}$ $<0)$ one finds an angle $\theta_{0}=2 \tan ^{-1} \sqrt{\eta_{R} /\left(P P^{\prime}-1\right)}$ where the thermoelectric power can change sign, i.e., a spin valve with noncollinear magnetic configuration can display a transition from electronlike to holelike transport. In a spin valve the thermally induced charge currents that enter the normal node from both sides can be made to cancel such that the net thermoelectric voltage vanishes, $\bar{S}=0$. Note that not only the individual spin currents but also the charge current depend on the effective spin polarizations. The MTP vanishes in the half-metallic limit $P=P^{\prime}= \pm 1$.

In the weakly thermalized regime on the other hand, the magnetothermopower [Eq. (29)] is twice as large, whereas the magnetoresistance and $\bar{S}(0)$ do not change (in the limit $\left.S^{2} \ll \mathcal{L}_{0}\right) . \bar{S}(\pi)$ is enhanced in this case because the heat spin accumulation facilitates the thermoelectric voltage buildup. In this regime an angular magnetothermopower is found even when $P_{S}=0$ provided that $\eta_{R}^{\prime} \equiv 2 \operatorname{Re} \partial_{\epsilon} G^{\uparrow \downarrow} / \partial_{\epsilon} G \neq \eta_{R}$, which is destroyed by full thermalization.

The Onsager-Kelvin relation between the total Seebeck and Peltier coefficients in spin valves, i.e., $\bar{\Pi}(\theta)=\bar{S}(\theta) T$ in which $\bar{\Pi} \equiv\left(-\dot{Q}_{c} / I_{c}\right)_{\Delta T=0}$, is found to hold in both thermalization regimes.

For a quantitative analysis we need to know more about the thermoelectric parameters for interfaces. In the absence of experimental estimates, we calculated the parameters from first principles within the framework of density functional theory for a number of interfaces which figure prominently in the field of magnetoelectronics. ${ }^{17}$ The values are given in Table I. For an $A \mid B$ interface, the calculation proceeds as follows. ${ }^{29,46}$ Self-consistent density functional theory calculations are first performed separately for bulk $A$ and $B$ materials. These calculations yield bulk charge and spin densities and potentials, and the corresponding Fermi energies. A selfconsistent interface calculation is next performed subject to the potentials (and densities) far from the interface being equal to their bulk values, up to a constant which is adjusted so as to equalize the Fermi energies. ${ }^{47}$ The interface breaks the lattice periodicity perpendicular to the interface leaving only two-dimensional periodicity parallel to the interface that is characterized by the two-dimensional Bloch vector $\mathbf{k}_{\|}$. The electronic structure of the localized perturbation formed by the interface is handled using a Green's-function method, a so-called "surface Green's function." The rank of the matrix of the perturbation is made finite and minimized by making use of the translational symmetry parallel to the interface and using a maximally localized basis of tight-binding (TB) muffin-tin orbitals (MTOs). ${ }^{48,49}$ To calculate the scattering matrix $\mathcal{S}$,

$$
\mathcal{S}\left(\mathbf{k}_{\|}, \epsilon\right) \equiv\left(\begin{array}{cc}
r\left(\mathbf{k}_{\|}, \epsilon\right) & t^{\prime}\left(\mathbf{k}_{\|}, \epsilon\right) \\
t\left(\mathbf{k}_{\|}, \epsilon\right) & r^{\prime}\left(\mathbf{k}_{\|}, \epsilon\right)
\end{array}\right),
$$

at real energies (at or close to the Fermi energy in the context of transport), we use a wave-function-matching scheme due to Ando ${ }^{50}$ which involves the calculation of individual scattering states far from the interface. The rank of the reflection and transmission matrices $r, r^{\prime}, t, t^{\prime}$ is determined by the number of Bloch states at a given energy $\epsilon$ and transverse wave vector $\mathbf{k}_{\|}$. The minimal TB-MTO basis is very efficient and makes it possible to model incommensurate lattices and various types of disorder using large lateral supercells..$^{29-51}$ Substitutional disorder where one or more layers of atoms form an alloy is conveniently treated by calculating the potentials self-consistently using a layer version ${ }^{47}$ of the coherent potential approximation ${ }^{52}$ and then distributing at random the site potentials in lateral supercells subject to maintenance of the appropriate layer concentrations. ${ }^{29}$ The mixing conductance is most easily calculated in terms of the reflection matrices. ${ }^{43,53} \mathrm{We}$ consider here interfaces in diffuse metallic systems which implies that we have to use a generalization of the Schep correction ${ }^{25,31}$ by replacing the bare $G^{\alpha \beta}(\epsilon)^{-1}$ with $G^{\alpha \beta}(\epsilon)^{-1}-\left[G_{\mathrm{N}}(\epsilon)^{-1}+\delta_{\alpha \beta} G_{\mathrm{F}}^{\alpha}(\epsilon)^{-1}\right]$, where $G_{\mathrm{N}}(\epsilon)$ $=e^{2} M_{\mathrm{N}}(\epsilon) / h$ and $G_{\mathrm{F}}^{\alpha}(\epsilon)=e^{2} M_{\mathrm{F}}^{\alpha}(\epsilon) / h$ are the single-spin Sharvin conductances of the normal and ferromagnetic metals forming the interface. The thermopower and other generalized thermoelectric parameters are determined by numerically differentiating the scattering matrix calculated as a function of the energy. Details of the numerical procedures will be given in a separate paper. ${ }^{30}$

We use the data in Table I to compute the angular dependence of the thermoelectric properties of a few spin valves for illustrative purposes. Spin-flip scattering is disregarded here but will be discussed in later sections. We plot the angular MTP for a $\mathrm{Fe}|\mathrm{Cr}| \mathrm{Fe}$ (001) spin valve with dirty inter- 


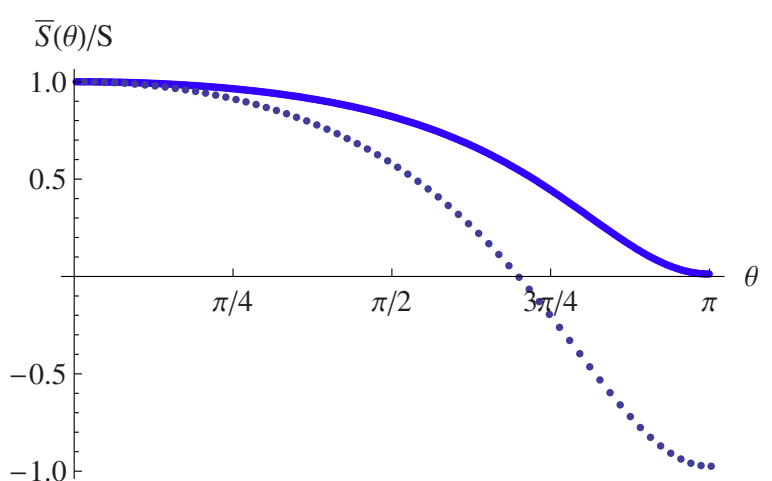

FIG. 2. (Color online) Magnetothermoelectric power as a function of relative angle between the magnetizations in a $\mathrm{Fe}|\mathrm{Cr}| \mathrm{Fe}$ (001) spin valve with dirty interfaces. The MTP is significantly different for the strong (full line) and weak (dotted line) thermalizations in the normal-metal spacer.

faces in Fig. 2 and for a $\mathrm{Co}|\mathrm{Cu}| \mathrm{Co}$ (110) spin valve with clean interfaces in Fig. 3. The angular MTP is enhanced in the weakly thermalized regime (shown by dotted lines) by up to a factor of two for the antiparallel configuration. Depending on $P$ and $P_{S}$, the MTP can be of any sign [see Eq. (29)].

We now turn to the Peltier effect of asymmetric spin valves. In the strongly thermalized regime and for $P_{S_{1}}=P_{S_{2}}$ $=0$, the Peltier cooling retains the simple form for normalmetal structures [Eq. (5)] whereas the total charge current is a complicated function of the magnetic configuration of the system. A magneto-Peltier effect (MPE), i.e., a dependence of the cooling power on the magnetic configuration, is found when the thermopower is spin dependent. For a voltagebiased spin valve with thermal asymmetry $S_{1} \neq S_{2}$ and $P_{1}^{\prime}$ $\neq P_{2}^{\prime}$, but $G_{1}=G_{2}=G, P_{1}=P_{2}=P$, and $S_{i}^{\uparrow()} \ll \sqrt{\mathcal{L}_{0}}$, we find for the temperature change of the normal-metal spacer

$$
\Delta T_{N}=\frac{\left(\gamma_{1}^{\mathrm{MP}} S_{1}-\gamma_{2}^{\mathrm{MP}} S_{2}\right) I_{c}}{2 \mathcal{L}_{0} G},
$$

where the spin-entropy factors,

$$
\gamma_{1(2)}^{\mathrm{MP}}(\theta)=\frac{\left(1-P_{1(2)}^{\prime} P\right) \tan ^{2} \theta / 2+\eta_{R}}{\left(1-P^{2}\right) \tan ^{2} \theta / 2+\eta_{R}}
$$

depend now on the magnetic configuration $\left(P_{1(2)}^{\prime} \neq P\right) . \gamma_{1(2)}^{\mathrm{MP}}$ $=1$ and $\left(1-P_{1(2)}^{\prime} P\right) /\left(1-P^{2}\right)$, respectively, for parallel and an-

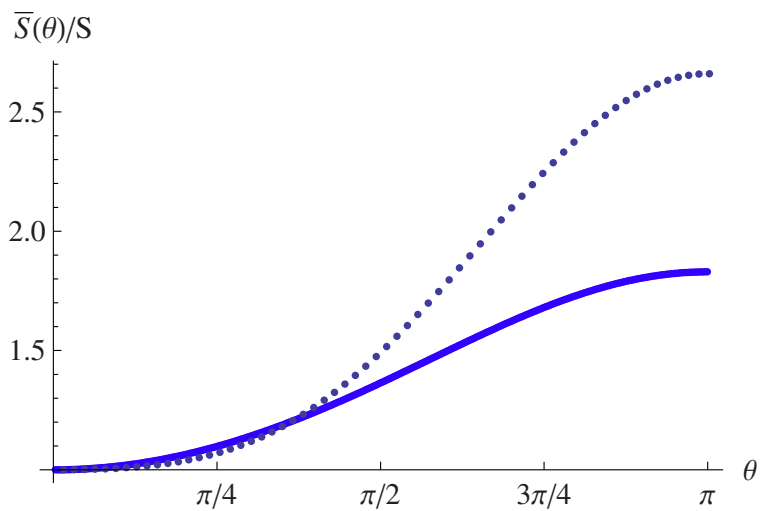

FIG. 3. (Color online) Same as Fig. 2 but for a $\mathrm{Co}|\mathrm{Cu}| \mathrm{Co}$ (110) spin valve with clean interfaces.

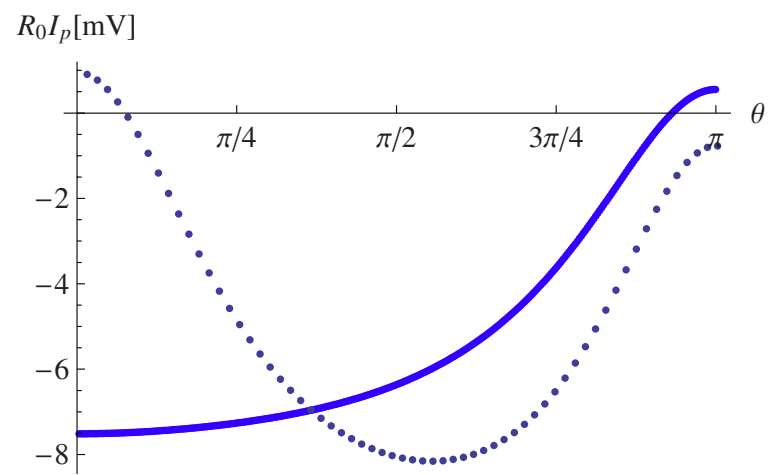

FIG. 4. (Color online) Magneto-Peltier cooling as a function of the angle between the magnetizations in the hypothetical bcc $\mathrm{Co}|\mathrm{Cr}| \mathrm{Fe}(001)$ spin valve. $R_{0} I_{p}(\theta)$ is the cooling power which compensates local Joule heating. The signal $R_{0}\left[I_{p}(\pi)-I_{p}(0)\right]$ is significantly different for strong (full line) and weak (dotted line) thermalizations in the normal-metal spacer.

tiparallel configurations. The MPE should therefore be observable in $R$ vs $I$ curves of spin valves during currentinduced magnetization reversal. According to Eq. (18) with $\bar{R}\left(I=I_{p}\right)=\bar{R}(I=0) \equiv R_{0}$, the temperature change $\Delta T_{N}=T_{N}(\theta)$ $-T_{0}$ corresponds to the cooling-power $R_{0} I_{p} \approx 2 \mathcal{L}_{0}\left(G_{1}\right.$ $\left.+G_{2}\right) T \Delta T_{N} / I_{p}$ such that

$$
R_{0}\left[I_{p}(\pi)-I_{p}(0)\right]=4 \frac{G_{1} P_{2}\left(P_{1}^{\prime}-P_{1}\right) \Pi_{1}-G_{2} P_{1}\left(P_{2}^{\prime}-P_{2}\right) \Pi_{2}}{G_{1}\left(1-P_{1}^{2}\right)+G_{2}\left(1-P_{2}^{2}\right)} .
$$

This signal contains unique information on the spin polarization of the thermopower. When thermalization is weak a MPE arises even when $P_{S_{1}}=P_{S_{2}}=0\left(P_{1(2)}^{\prime}=P_{1(2)}\right)$. A sign change in the cooling power is also expected. In the strongly thermalized regime this arises from different angular dependences of the spin-entropy factors. When the effective thermopowers are equal, $\gamma_{1}^{\mathrm{MP}}\left(\theta_{0}\right) S_{1}=\gamma_{2}^{\mathrm{MP}}\left(\theta_{0}\right) S_{2}$, no Peltier cooling is expected, $R_{0} I_{p}\left(\theta_{0}\right)=0$.

In Figs. 4 and 5, we illustrate the magneto-Peltier cooling by computing the angular-dependent cooling power $R_{0} I_{p}(\theta)$, at room temperature, for a hypothetical bcc $\mathrm{Co}|\mathrm{Cr}| \mathrm{Fe}(001)$ spin-valve structure with clean interfaces and for an asymmetric $\mathrm{Co}|\mathrm{Cu}| \mathrm{Co}(001)$ with one ideal and one disordered

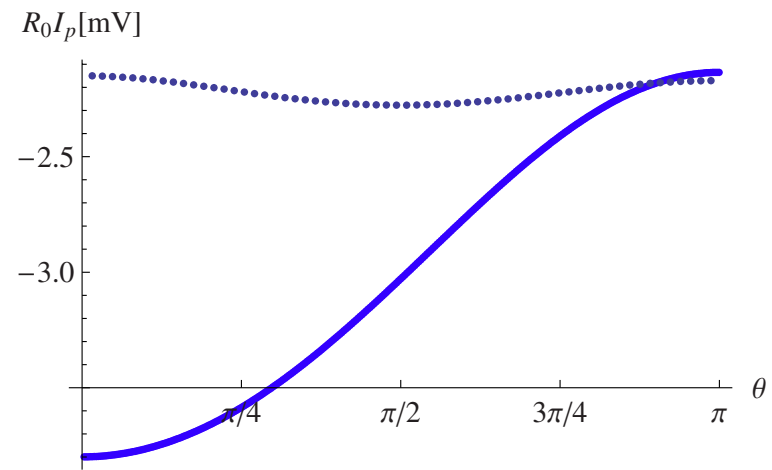

FIG. 5. (Color online) Same as Fig. 4 but for an asymmetric $\mathrm{Co}|\mathrm{Cu}| \mathrm{Co}(001)$ spin valve with one clean and one disordered interface. 
interface, using parameters from Table I. For comparison, we include the results in the weakly thermalized regime, depicted in the figures by dotted lines. The dependence of the Peltier cooling on the magnetic configuration observed in Fig. 4 for the $\mathrm{Co}|\mathrm{Cr}| \mathrm{Fe}$ spin valve is caused by the relatively large values of the interface thermopower parameters $P$ and $P^{\prime}$. The relatively weak magneto-Peltier signal, $R_{0}\left[I_{p}(\pi)\right.$ $\left.-I_{p}(0)\right]$ vanishes when $G_{1}=G_{2}$ and $P_{1}=P_{2}$, for weakly thermalized electrons (dotted line) is strongly enhanced by the interspin energy exchange in the opposite limit. The MPE for the asymmetric $\mathrm{Co}|\mathrm{Cu}| \mathrm{Co}$ (001) structure (Fig. 5) displays similar effect but with smaller amplitudes. For the strongly thermalized electron case, the MPE caused by interface scattering, $R_{0}\left[I_{p}(\pi)-I_{p}(0)\right]$, is on the order of $1-10 \mathrm{mV}$, which is smaller than the experimental values ${ }^{18,19} R_{0} I_{p} \approx 20-40 \mathrm{mV}$. For a typical current density of $10^{8} \mathrm{~A} / \mathrm{cm}^{2}$, we find a maximum temperature drop $\Delta T_{N}(\theta) \approx 0.15 \mathrm{~K}$ which is also too small to explain experiments. Note that in the above we have assumed that interface scattering is dominant. As explained in the next sections, we show that these numbers are increased by including scattering in the bulk.

The magneto-Peltier effect vanishes for symmetric spinvalve structures. Introducing the asymmetry $G_{1} \neq G_{2}$, we obtain an angle-dependent temperature modulation,

$$
\begin{aligned}
\mathcal{L}_{0} \Delta T_{N}(\theta) & \\
= & \frac{2\left(G_{1}-G_{2}\right) P\left(P^{\prime}-P\right) S I_{c} \sin ^{2} \frac{\theta}{2}}{\left(G_{1}+G_{2}\right)^{2}\left(1-P^{2}\right) \eta_{R}+G_{1} G_{2}\left(1-P^{2}-\eta_{R}\right)^{2} \sin ^{2} \theta},
\end{aligned}
$$

even for equal thermopowers and spin polarizations at the interfaces. Analytical expressions for the Peltier cooling in the weak thermalization regime, in which the spin-mixing thermopower $\left(\eta_{R(I)}^{\prime}\right.$ in Table I) becomes a relevant parameter, are much more complex. The computation is straightforward, however, and is easily carried out when the necessity arises.

\section{SPIN-CONSERVING BULK IMPURITY SCATTERING}

In this section we discuss the contribution of bulk scattering for the case of wires with constant cross section $A_{0}$, first for nonmagnetic metals and then for magnetic structures, both in the strongly thermalized limit. A normal-metal pillar is a heterostructure $\mathcal{R}_{A}\left|N_{A}\left(L_{A}\right)\right| N(L)\left|N_{B}\left(L_{B}\right)\right| \mathcal{R}_{B}$, where $N_{i}\left(L_{i}\right)$ denotes a layer of material $i(=A, B)$ with thickness $L_{i}$ that can be larger than the elastic mean-free path due to disorder scattering. The length $L$ of the central island $N$ is so short that its (bulk) resistance can be disregarded. The external reservoirs $\mathcal{R}_{A(B)}$ are in thermal equilibrium but at different temperatures and/or voltages. The spreading resistance at an abrupt opening can be accounted for by an effective length parameter. ${ }^{20}$ The electron distribution functions in the disordered metal wires follow from the diffusion equation in the bulk and are connected at the interfaces by (quantum mechanical) boundary conditions. ${ }^{25}$ The conserved particle/ heat currents can be obtained from Eq. (4) by replacing
$-\left(V_{1(2)}-V_{N}\right)$ with $L_{A(B)} \nabla \mu_{A(B)} / e, T_{1(2)}-T_{N}$ with $L_{A(B)} \nabla T_{A(B)}$ and the interface conductance [Eq. (2)] with the electric conductivity $\sigma_{A(B)}=e^{2} \mathcal{N}_{A(B)} D_{A(B)}$, where $\mathcal{N}$ and $D$ are energydependent densities of states and diffusion constants of the bulk materials, respectively. Mott's formula, $S_{A(B)}=$ $-\left.e \mathcal{L}_{0} T \partial_{\epsilon} \ln \sigma_{A(B)}\right|_{\epsilon_{F}}$, holds for the diffusion thermopower which usually dominates at high temperatures. ${ }^{54,55}$ In linear response charge and energy current conservations imply $\nabla^{2} \mu=0$ and $\nabla^{2} T=0$. The chemical potential and temperature depend linearly on position except for jumps at the contacts which are governed by the interface parameters. The local chemical potential and temperature are then found by the charge and energy current conservations at the boundaries.

As a function of the applied electric current we obtain the following expression for the temperature change $\Delta T_{N}$ on the normal-metal island:

$$
\Delta T_{N}=\left(\frac{S_{A}-S_{B}}{G_{A} G_{B}}+\frac{S_{1}-S_{2}}{G_{1} G_{2}}+\frac{S_{A}-S_{2}}{G_{A} G_{2}}+\frac{S_{1}-S_{B}}{G_{1} G_{B}}\right) \frac{G_{\mathrm{tot}} I}{\mathcal{L}_{0}},
$$

where $G_{A(B)}=\sigma_{A(B)} A_{0} / L_{A(B)}$ and $S_{A(B)}$ are the bulk (Drude) conductances and thermopowers in the leads; $G_{\text {tot }}^{-1}$ $=\sum_{i=1,2, A, B} G_{i}^{-1}$ is the total series conductance. The interface contribution to the Peltier cooling disappears when $S_{1}=S_{2}$ and $G_{1} / G_{2}=G_{A} / G_{B}$. When $S_{A}=S_{B}$ and $\sigma_{A}=\sigma_{B}$, Peltier cooling is possible for different lengths of the normal leads $\left(L_{A} \neq L_{B}\right) .{ }^{56}$ Equation (35) can be simplified by introducing the lumped conductances $G_{L}=G_{A} G_{1} /\left(G_{A}+G_{1}\right)$ and $G_{R}$ $=G_{2} G_{B} /\left(G_{2}+G_{B}\right)$ as well as the thermopowers $S_{L}$ and $S_{R}$ with $S_{L} / G_{L}=S_{A} / G_{A}+S_{1} / G_{1}$ and $S_{R} / G_{R}=S_{2} / G_{2}+S_{B} / G_{B}$ for the left and right parts of the normal island. In terms of the new parameters we find

$$
\Delta T_{N}=\frac{\left(S_{L}-S_{R}\right) I}{\mathcal{L}_{0}\left(G_{L}+G_{R}\right)},
$$

which, as expected, has the same form as Eq. (5) in the limit $S^{2} \ll \mathcal{L}_{0}$.

Replacing the normal lead $N_{A}$ by a magnetic lead, say, $F_{A}$, we find that the thermoelectric cooling obeys Eq. (23) after replacing the interface conductances and thermopowers $G_{1(2)}$ and $S_{1(2)}$ with $G_{L(R)}$ and $S_{L(R)}$, provided that the spin polarizations in bulk layers and contacts are the same. A more complicated structure such as $\mathrm{Co}|\mathrm{Au}| \mathrm{Ti} \mid \mathrm{Au}$ can be shown to be equivalent to an $\mathrm{F}|\mathrm{N}| \mathrm{N}_{2}$ pillar by a similar lumping of parameters.

We now turn to the MPE, i.e., the dependence of the Peltier cooling on the magnetic configuration of a spin-valve structure, in the presence of bulk scattering. A simple analytical expression for the cooling power (or the local Joule heating compensation current $I_{p}$ ) can be obtained when the spin polarizations of the bulk and interfaces are equal,

$$
R_{0} I_{p}=2\left(\gamma_{L}^{\mathrm{MP}} \Pi_{L}-\gamma_{R}^{\mathrm{MP}} \Pi_{R}\right)
$$

where the spin-entropy factors $\gamma_{L(R)}^{\mathrm{MP}}$ are 


$$
\gamma_{L}^{\mathrm{MP}}=\frac{G_{L}\left[1-P_{A} P_{B}-\left(P_{A}-P_{B}\right) P_{A}^{\prime}\right]+G_{R}\left(1-P_{B}^{2}\right)}{G_{L}\left(1-P_{A}^{2}\right)+G_{R}\left(1-P_{B}^{2}\right)} .
$$

An expression for $\gamma_{R}^{\mathrm{MP}}$ is obtained by interchanging the indices $L \leftrightarrow R$ and $A \leftrightarrow B$. Equations (37) and (38) reduce to Eq. (33) when bulk scattering is disregarded.

A phonon (or magnon) thermal current can transfer momentum to the electrons in the presence of inelastic scattering which in turn generates an additional electric field and modifies the thermopower. For normal (as well as ferromagnetic) metals at sufficiently low temperatures a contribution of the phonon- (and magnon-) drag effect may become significant. ${ }^{57,58}$ The magnon-drag effect is likely to be suppressed strongly in heterostructures since magnons cannot escape the ferromagnets. Strong phonon scattering at interfaces will likewise reduce the phonon-drag effect in multilayers. A microscopic treatment of the phonon-drag effect in heterostructures is beyond the scope of the present paper, however. At elevated temperatures, where the drag effect can be disregarded, Mott's formula holds approximately even in the presence of inelastic scattering..$^{59,60}$

\section{SPIN-FLIP BULK IMPURITY SCATTERING}

Here we study the influence of spin-flip relaxation on the Peltier and Seebeck effects in magnetic $\mathcal{R}_{A}\left|F_{A}\left(L_{A}\right)\right| N(L)\left|F_{B}\left(L_{B}\right)\right| \mathcal{R}_{B}$ nanopillars, where $F_{A}$ and $F_{B}$ denote disordered ferromagnetic layers, with collinear magnetization directions. We assume that bulk impurity scattering is dominant so that interfaces may be disregarded. The charge and spin distribution functions in the ferromagnet, $f_{c(s)}=\left(f^{\uparrow} \pm f^{\downarrow}\right) / 2$, respectively, are then solutions of the spindiffusion equations that are continuous at the interfaces. ${ }^{61} \mathrm{In}$ the strongly thermalized regime, defining $\mu_{s}=\mu_{\uparrow}-\mu_{\downarrow}$ and $\mu_{c}=\left(\mu_{\uparrow}+\mu_{\downarrow}\right) / 2$ as, respectively, the spin and charge chemical potentials, we find (see Appendix B for details) the following thermoelectric spin-diffusion equations in a ferromagnet

$$
\begin{gathered}
\nabla^{2} \mu_{s}=\frac{\mu_{s}}{l_{\mathrm{sf}}^{2}}, \\
\nabla^{2} \mu_{c}=-P \frac{\mu_{s}}{2 l_{\mathrm{sf}}^{2}}, \\
\nabla^{2} T=\frac{\left(P^{\prime}-P\right) S}{\mathcal{L}_{0}} \frac{\mu_{s}}{2 l_{\mathrm{sf}}^{2}} .
\end{gathered}
$$

Here $l_{\text {sf }}$ stands for the spin-flip diffusion length and $P$ and $P^{\prime}$ are the spin polarizations of the bulk conductivity and its energy derivative in the ferromagnet. These equations have to be solved with continuity boundary conditions at the interfaces. The expressions for the currents are similar to Eq. (19) after replacing temperature and voltage differences by gradients and conductances by conductivities. Equation (41) is required by the conservation of charge and energy currents. According to this equation the decay of the spin accumulation in the ferromagnet provides a source or sink of heat

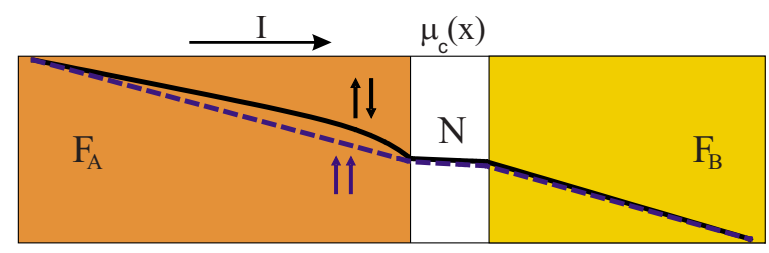

(a)

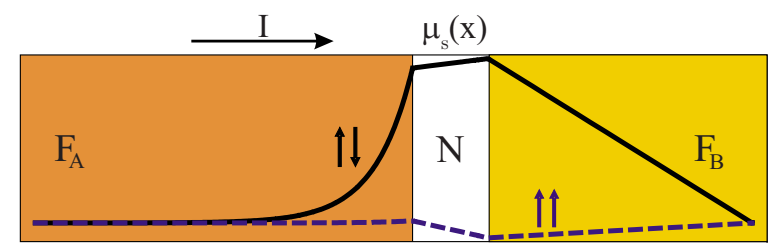

(b)

FIG. 6. (Color online) Illustration of the local charge and spin chemical potentials in a $\mathrm{F}_{A}|\mathrm{~N}| \mathrm{F}_{B}$ spin valve biased by a voltage difference for both parallel $(\uparrow \uparrow)$ and antiparallel $(\uparrow \downarrow)$ alignments of the magnetizations. $\mathrm{F}_{B}\left(P=0.8, \lambda_{B}=0.1, S_{B}=-1 \mu \mathrm{V} / \mathrm{K}\right)$ has been chosen to have weak spin-flip scattering and thermopower compared with $\mathrm{F}_{A}\left(P=0.8, \lambda_{A}=10, S_{A}=-20 \mu \mathrm{V} / \mathrm{K}\right)$. The thin normalmetal spacer is chosen to be highly conductive $\left(\rho_{N} \ll \rho_{A(B)}\right.$ $=10 \mu \Omega \mathrm{cm}$ ) and to have a thermopower equal to that of $\mathrm{F}_{B}$. Note that spin accumulation is assumed to vanish at the two ends (at reservoirs).

currents (when $P^{\prime} \neq P$ ). We can understand this effect by the charge accumulation that is locally generated by spin flips in ferromagnets [Eq. (40)]. Similarly, spin-flip scattering in the presence of spin polarization of thermopower modifies the distribution functions in a way that can be interpreted as a source or sink of heat as expressed in Eq. (41). The spatial variation in $\mu_{c(s)}(x)$ and $T(x)$ in a voltage-biased spin valve is sketched in Figs. 6 and 7. The local charge and spin chemical potentials in a spin valve biased with a voltage difference

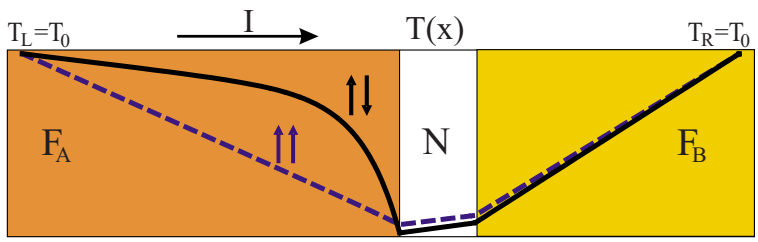

(a)

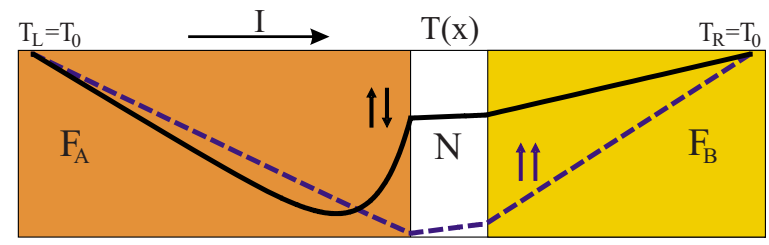

(b)

FIG. 7. (Color online) Illustration of local temperature distribution in the voltage-biased $\mathrm{F}_{A}|\mathrm{~N}| \mathrm{F}_{B}$ spin-valve structure as in Fig. 6 for both parallel $(\uparrow \uparrow)$ and antiparallel $(\uparrow \downarrow)$ alignments of the magnetizations. The different temperature profiles correspond to (a) $P^{\prime} \ll P\left(P_{S}=-0.8\right)$ and (b) $P^{\prime} \gg P\left(P_{S}=-1.8\right)$. For the parallel alignment of the magnetizations the Peltier cooling is insensitive to spin flips and reaches its maximum in the central node. 
(Fig. 6) do not depend much on the thermopower or the Peltier cooling. More interesting are the results in Fig. 7, illustrating the strong dependence of the local temperature on magnetization configuration, the strength of the spin-flip scattering, and the spin polarization of the thermopower.

In the following we assume identical spin polarization and spin-flip diffusion length $l_{\mathrm{sf}}$ in the magnetic leads and $l_{\mathrm{sf}}^{N}$ $\gg L$. Our results become simple when the only asymmetries of the pillar are $\sigma_{A} \neq \sigma_{B}, S_{A} \neq S_{B}$. For parallel alignments of the magnetizations the Peltier cooling is equal to that of a normal-metal structure with $R_{0} I_{p}(0) \approx 2 \mathcal{L}_{0}\left(G_{A}+G_{B}\right) T \Delta T_{N} /$ $I_{p}=2\left(\Pi_{A}-\Pi_{B}\right)$. However, the magneto-Peltier signal in the presence of spin decay becomes

$$
R_{0}\left[I_{p}(\pi)-I_{p}(0)\right]=-\frac{\tanh \lambda}{\lambda} \frac{4 P P_{S}}{1+P P_{S}} \frac{G_{A} \Pi_{A}-G_{B} \Pi_{B}}{G_{A}+G_{B}},
$$

where $\lambda=L_{A} / l_{\mathrm{sf}}^{A}=L_{B} / l_{\mathrm{sf}}^{B}$ is a measure of the spin-flip scattering in the ferromagnets. The magneto-Peltier signal decays with increasing $\lambda$, e.g., on using a thicker magnetic leads. The magneto-Peltier signal vanishes when $\lambda \gg 1$ and reduces to an expression equivalent to Eq. (33) in the opposite limit. Spin flips in the normal-metal spacer (with thickness comparable to or longer than $l_{\mathrm{sf}}^{N}$ ) also reduce the magneto-Peltier signal.

For the spin-valve structure in the presence of bulk spin diffusion in the ferromagnets, we find the following results for the magnetoresistance MR as well as the magnetothermopower MTP. For the parallel configuration, as expected, no spin-flip contribution to the total resistance and thermopower is obtained i.e., $R_{P}=R_{A}+R_{B}$ and $S_{P}=\left(R_{A} S_{A}\right.$ $\left.+R_{B} S_{B}\right) /\left(R_{A}+R_{B}\right)$. However, for the antiparallel configuration we find

$$
\begin{gathered}
R_{A P}-R_{P}=\frac{\tanh \lambda}{\lambda} \frac{4 P^{2}}{1-P^{2}} \frac{R_{A} R_{B}}{R_{A}+R_{B}}, \\
S_{A P}-S_{P}=-\frac{\tanh \lambda}{\lambda} \frac{2 P P_{S}}{1+P P_{S}} \frac{R_{A} R_{B}}{\left(R_{A}+R_{B}\right)^{2}}\left(S_{A}+S_{B}\right)
\end{gathered}
$$

The MTP is therefore proportional to the giant magnetoresistance, independent of the spin-flip scattering strength $\lambda$. In contrast to the MR (at $\lambda \gg 1$ ) it is not possible to model spin-flip scattering for magnetothermoelectric effects by replacing $R_{A(B)}$ by the resistance of the magnetically active region $\lambda^{-1} R_{A(B)}$.

\section{RELEVANCE FOR EXPERIMENTS}

In the magnetic nanopillars considered by Fukushima et $a l{ }^{18,19}$ the magnetic or normal leads that connect the central spacer to the wide external reservoirs are so long that the bulk scattering is important: a 100-nm-long cobalt wire has a resistance $\rho_{\mathrm{Co}} L \simeq 6 \mathrm{f} \Omega \mathrm{m}^{2}$ at room temperature, which is larger than the interface resistance $R_{\mathrm{Co} \mid \mathrm{Cu}} \approx 0.25 \mathrm{f} \Omega \mathrm{m}^{2}$ (see Table I). The effective thermopower $S_{L} \approx S_{\mathrm{Co}_{0}} \simeq-31 \mu \mathrm{V} / \mathrm{K}$ from the bulk scattering is also larger than the interface thermopower $S_{\mathrm{Co} \mid \mathrm{Cu}} \approx-6 \mu \mathrm{V} / \mathrm{K}$. The interface contribution to the total thermopower would become more important for high-resistance interfaces, such as tunneling barriers or point contacts, or structures with thinner layers.

The lattice exchanges energy with the conduction electrons by inelastic electron-phonon interactions. In principle, there is a net heat current flowing between the electron system and the lattice/substrate. In a steady state situation it is reasonable to assume that electron and lattice temperatures are identical and resistance changes reflect the electron temperature. When Peltier cooling and Joule electron heating compensate each other the temperature change vanishes. Estimating the nonlinear electron heating in the island by $\mathcal{L}_{0} T \Delta T_{N}=G_{\text {tot }}^{-1} I^{2} / 2\left(G_{L}+G_{R}\right)$ and using Eq. (23) in terms of the lumped conductances and thermopowers, the compensation current $I_{p}$ or cooling power $R_{0} I_{p}$ for $\mathrm{F}_{A}|\mathrm{~N}| \mathrm{N}_{B}$ structures such as $\mathrm{Co}|\mathrm{Cu}| \mathrm{Au}$ nanopillars can be expressed as

$$
R_{0} I_{p} \approx 2\left(\gamma_{L} \Pi_{L}-\Pi_{R}\right) .
$$

Such an expression holds as well for $\mathrm{Co}|\mathrm{Cu}| \mathrm{Co} \mid \mathrm{Au}$ structures when the magneto-Peltier effect for the symmetric part $\mathrm{Co}|\mathrm{Cu}| \mathrm{Co}$ can be disregarded. ${ }^{18,19}$ The factor of 2 difference with Refs. 18 and 19 has been noted already above. We find below that including this factor leads to a better agreement of a simple model of bulk thermopowers with experiments. Also the too-large pillar cross sections with which Gravier et $a l .{ }^{20}$ fitted their numerical results to the experiments can be traced back to this factor 2 in the Joule heating. Our model might not be appropriate for the samples of Fukushima et $a l .{ }^{18,19}$ that contain a highly resistant, presumably oxide, layer over which much of the voltage drop occurs. Such a layer, when sufficiently thick, might be better described as a bulk resistor in which Joule heat is preferentially generated. At the compensation current $I_{p}$, finite temperature variation profiles may persist since the Joule and Peltier sources are spatially separated. A simulation beyond our simple model might then be required for a quantitative description.

Experimental values of $R_{0} I_{p}$ for Co $\mid$ Au nanopillars can be read off the figures published by different groups, amounting to (in $\mathrm{mV}$ ) $19,{ }^{62} 23.0,{ }^{63}$ and 22.5. ${ }^{18,19}$ These numbers agree well with the following results. For a finite length of the bulk layers, i.e., $L_{\mathrm{Co}}=60 \mathrm{~nm}$ and $L_{\mathrm{Au}}=120 \mathrm{~nm}$ and taking into account the interface scattering, when disregarding the spin polarization of the thermopower $P_{S}$ we find $R_{0} I_{p}=19.5 \mathrm{mV}$. Here we also assumed $G_{R} \ll G_{L}$ caused by an oxide layer on the nonmagnetic side of the structure, $S_{\mathrm{Cu} \mid \mathrm{Au}} \simeq 0, S_{\mathrm{Co} \mid \mathrm{Cu}} \simeq$ $-6 \mu \mathrm{V} / \mathrm{K}$ and used the bulk parameters from Ref. 20. $R_{0} I_{p} \approx 2\left(\Pi_{\mathrm{Co}}-\Pi_{\mathrm{Au}}\right)=19.6 \mathrm{mV}$ indicates that the Peltier cooling is not significantly affected by interface scattering. A finite $P_{S}$ can enlarge or reduce the above estimates. The spinentropy coupling factor $\gamma_{L} \approx\left(1-P^{\prime} P\right) /\left(1-P^{2}\right)$ when the bulk and interface spin polarizations are the same. For Co we took $P=0.44 .{ }^{64}$ Conflicting values $P_{S}=-0.18<0\left(S^{\uparrow}=0.7 S_{\downarrow}\right)$ (Refs. 13 and 14) and $P_{S}=+0.42>0\left(S^{\uparrow}=-30 \mu \mathrm{V} / \mathrm{K}, S_{\downarrow}=\right.$ $-12 \mu \mathrm{V} / \mathrm{K}$ ) (Ref. 38) are found in the literature. According to Table $\mathrm{I}, P_{S}$ of the $\mathrm{Co} \mid \mathrm{Cu}$ interface can also have either sign. The two values for $P_{S}$ modify the above estimate to $R_{0} I_{p}=21$ and $17 \mathrm{mV}$, respectively, possibly favoring a $P_{S}$ $<0$ when compared with the observed values. The adiabatic spin-entropy expansion term $\left(k_{B} T \ln 2\right) I / e$ considered in Ref. 
21 is in our opinion an extrapolation of a concept from equilibrium thermodynamics that does not play a role in the current-induced (nonequilibrium) Peltier cooling.

We proceed by estimating the magnitude of the temperature drop that can be realized by the Peltier effect in the magnetic heterostructure $\mathrm{Co}(60 \mathrm{~nm})|\mathrm{Au}(20 \mathrm{~nm})| \mathrm{Cr}(120 \mathrm{~nm}) .{ }^{20}$ At room temperature the bulk thermopowers of both $\mathrm{Co}$ and $\mathrm{Cr}$ are relatively large and have opposite signs $\left(S_{\mathrm{Cr}}=+21.56 \mu \mathrm{V} / \mathrm{K}\right)$. The temperature drop $\Delta T_{\mathrm{Au}} \approx I_{p}\left(S_{L}-S_{R}\right) /\left(\mathcal{L}_{0}\left(G_{L}+G_{R}\right)\right)$ in the central island amounts to $4.8 \mathrm{~K}$ at $I_{p}=10 \mathrm{~mA}$ for a cross section of $70 \times 200 \mathrm{~nm}$, at a current density of $\sim 10^{8} \mathrm{~A} / \mathrm{cm}^{2}$, which is close to the maximum temperature drop in the temperature profiles computed in Ref. 20 (we find a cooling power $R_{0} I_{p}$ $=30 \mathrm{mV}$, which is smaller than the observed value of 41 $\mathrm{mV}$, however). The temperature reduction per unit of electric current is sensitive to the thickness of the leads. For the thick magnetic layers $\Delta T_{\mathrm{Au}} \approx I_{p}\left(S_{\mathrm{Co}}-S_{\mathrm{Cr}}\right) / \mathcal{L}_{0}\left(G_{\mathrm{Co}}+G_{\mathrm{Cr}}\right)$. Spin polarization of the thermopower in Co can modify the amount of the temperature reduction, up to $8 \%$ for $\left|P_{S}\right|=0.4$.

In the experiments of Fukushima et al. ${ }^{18,19}$ the leads connected to the external reservoirs are long compared to the spin-flip diffusion length. In that regime a magneto-Peltier effect should be small. Let us therefore consider a spin-valve structure such as $\mathrm{Co}_{001}(10 \mathrm{~nm})|\mathrm{Au}(20 \mathrm{~nm})| \mathrm{Co}_{001}(5 \mathrm{~nm})$ in which spin-flip scattering is less important. Due to the different lengths of the bulk Co layers the Peltier cooling does not vanish at this structure even for a parallel magnetic configuration; recall for example Eq. (34) when $G_{1} \neq G_{2}$. For the parallel alignment of the magnetizations with $P_{A}=P_{B}$ one finds $\gamma_{L(R)}^{\mathrm{MP}}=1$, equivalent to a normal-metal structure, whereas the spin-entropy coupling parameter differs for the antiparallel magnetic configuration when $P_{A}=-P_{B}$. Let us now consider a small $G_{1}=0.01 G_{2}$, e.g., caused by an oxide layer at the junction between the thick Co layer and the normal-metal spacer, and use typical data in Table I for the interface scattering (at room temperature). Adopting bulk values $P_{S}=-1.18$ and $P_{S}=0.42$, we find, respectively, the magneto-Peltier signals $R_{0}\left[I_{p}(\pi)-I_{p}(0)\right]=-1.59$ and +6.7 mV, which should be experimentally observable. Replacing the bulk parameters of the thicker Co layer with $\rho_{\mathrm{Fe}}=9.7 \times 10^{-8} \Omega \mathrm{m}$ and $S_{\mathrm{Fe}}=+20 \mu \mathrm{V} / \mathrm{K}$, the Peltier cooling is increased and the magneto-Peltier signals read as $R_{0}\left[I_{p}(\pi)-I_{p}(0)\right]=+3.2$ and $-2 \mathrm{mV}$. Finally we mention that the magneto-Peltier cooling via the bulk scattering can be also sensitive to the degree of energy relaxation, but discussion of the details is beyond the scope of the present paper.

Since the thermopower-to-conductance ratios $S_{i} / G_{i}$ of the intermetallic interfaces studied up to now are smaller than the bulk values for thicker magnetic layers, for the material combinations considered above we do not expect an increased cooling power by reducing the thickness of the nanopillars to the interface-dominated regime. The interface contributions are important for (classical) point contacts or pinholes in thick tunneling barriers since $S_{I}$ can remain unmodified while $G_{I}$ is strongly reduced. Magnetic tunnel junctions are interesting subjects for magneto-Peltier studies since much higher $S / G$ ratios can be expected.

The spin-Seebeck effect ${ }^{65}$ recently observed in a very long ferromagnetic metal appears to have a different origin than the conventional mechanisms of spin and heat diffusion. The observed thermoelectric spin signal parametrized by a spin-Seebeck coefficient $\left(S_{s}=-2 \mathrm{nV} / \mathrm{K}\right.$ at room temperature ${ }^{65}$ ) is much smaller than both the interface and bulk thermopowers considered above. We therefore do not expect that the spin-Seebeck effect would significantly modify our findings.

\section{SUMMARY AND CONCLUSIONS}

We studied the Peltier effect in nanoscale metallic multilayer structures involving ferromagnets using a semiclassical theory of thermoelectric transport in magnetic heterostructures including the spin relaxations and the effects of electron interactions in limiting cases. The Peltier cooling/ heating depends in general on the spin degree of freedom as a function of spin and energy-dependent bulk and interface scattering. We predict a magneto-Peltier effect in spin valves, i.e., a dependence of Peltier cooling on the relative alignment of the two magnetization directions, that can arise from the spin polarization of thermopowers and is sensitive to the spin-flip scattering as well as strength of the inelastic collisions in the normal-metal spacer. Similar behavior is found for the magnetothermopower which might be even easier to observe in experiments (when thermoelectric voltage is measured rather than temperature). For ferromagnetic layers with thickness on the order or smaller than the spin-flip diffusion length, the magneto-Peltier effect should be observable in terms of magnetic-field-dependent resistance shifts in the $R(I)$ characteristics, i.e., the cooling power. Estimates for the Peltier cooling based on our model and available parameters agree relatively well with experiments as well as numerical models in which the bulk scattering dominates.

\section{ACKNOWLEDGMENTS}

We thank J. Bass, A. Brataas, T. Heikkila, S. Maekawa, Y. V. Nazarov, S. Takahashi, and J. Xiao for helpful discussions. This work is supported by "NanoNed," a nanotechnology program of the Dutch Ministry of Economic Affairs. It is also part of the research program for the "Stichting voor Fundamenteel Onderzoek der Materie" (FOM), and the use of supercomputer facilities was sponsored by the "Stichting Nationale Computer Faciliteiten" (NCF), both financially supported by the "Nederlandse Organisatie voor Wetenschappelijk Onderzoek" (NWO).

\section{APPENDIX A: PHONONS}

The Peltier effect in the presence of phonon heat conduction and electron-phonon interactions can be modeled in linear response as follows: the net heat current flowing between the electron and phonon subsystems of the island for small temperature differences $T_{e}^{N}-T_{p}^{N} \ll T_{p}^{N}$ may be parametrized by the simple linear equation $\dot{Q}_{e-p}=-\kappa_{e-p}\left(T_{e}^{N}-T_{p}^{N}\right)$. ${ }^{66}$ For a phonon temperature drop of $\Delta T_{p}$ across an interface, $\dot{Q}_{p}=$ $-\kappa_{p} \Delta T_{p}$ with $\kappa_{p}$ the phonon thermal conductance of the junction. ${ }^{34}$ The energy conservation laws then read as $\dot{Q}_{e}$ $+\dot{Q}_{e 2}+\dot{Q}_{e-p}=0$ and $\dot{Q}_{p 1}+\dot{Q}_{p 2}-\dot{Q}_{e-p}=0$ for the electron and 
phonon subsystems, respectively. The electron temperature in the node $[$ Eq. (5)] is then modified as follows:

$$
\Delta T_{e}^{N}=\frac{\left(\Pi_{1}-\Pi_{2}\right) I}{\kappa_{e 1}+\kappa_{e 2}+\gamma_{p}\left(\kappa_{p 1}+\kappa_{p 2}\right)}
$$

and $\Delta T_{p}^{N}=\gamma_{p} \Delta T_{e}^{N}$ where $\gamma_{p}=\kappa_{e-p} /\left(\kappa_{p 1}+\kappa_{p 2}+\kappa_{e-p}\right)$. In the limit $\kappa_{p 1(2)} \ll \kappa_{e-p}$ the Peltier cooling is reduced by the sum of the total thermal conductances $\kappa_{1(2)}=\kappa_{e 1(2)}+\kappa_{p 1(2)}$. The figure of merit $S \Delta T_{e}^{N} / \Delta V$ is then further decreased by taking into account the contribution of the phonon heat conduction $\left(\dot{Q}_{p} \neq 0\right)$.

\section{APPENDIX B: THERMOELECTRIC SPIN-DIFFUSION EQUATIONS}

In a diffusive magnetic metal in the steady state the Boltzmann transport equation in the relaxation time approximation leads to the following spectral spin-diffusion equations for the local variation of the spin distribution functions $f^{\uparrow(\downarrow)}(\epsilon)$ for each spin $\alpha$ as

$$
\nabla^{2} f^{\alpha}(\epsilon)=\frac{f^{\alpha}(\epsilon)-f^{-\alpha}(\epsilon)}{\left(l_{\mathrm{sf}}^{\alpha}\right)^{2}},
$$

where $l_{\mathrm{sf}}^{\alpha}=\sqrt{D^{\alpha} \tau_{\mathrm{sf}}^{\alpha}}$ are the spin-dependent diffusion lengths. Under the detailed balance condition $\mathcal{N}^{\uparrow} / \tau_{\text {sf }}^{\uparrow}=\mathcal{N} \downarrow / \tau_{\text {sf }}^{\downarrow}$ the spectral spin-diffusion equations can be rewritten as

$$
\nabla^{2} f_{s}(\epsilon)=\frac{f_{s}(\epsilon)}{l_{\mathrm{sf}}^{2}},
$$

$$
\nabla^{2} f_{c}(\epsilon)=-P \frac{f_{s}(\epsilon)}{2 l_{\mathrm{sf}}^{2}} .
$$

where $\left(l_{\mathrm{sf}}\right)^{-2}=\left(l_{\mathrm{sf}}^{\uparrow}\right)^{-2}+\left(l_{\mathrm{sf}}^{\downarrow}\right)^{-2}$ and the charge and spin distribution functions, $f_{c(s)}=\left(f^{\uparrow} \pm f^{\downarrow}\right) / 2$, have been introduced. In the strongly thermalized regime the spin-diffusion equations can be expressed in terms of the spin chemical potentials $\mu_{\uparrow(\downarrow)}=\left(\mu_{c} \pm \mu_{s} / 2\right)$ and the electron temperature $T$. After inserting the linear expansions,

$$
\begin{gathered}
f_{s}(\epsilon) \approx\left(-\frac{\partial f_{0}}{\partial \epsilon}\right) \mu_{s}, \\
\nabla^{2} f_{c(s)}(\epsilon) \approx\left(-\frac{\partial f_{0}}{\partial \epsilon}\right)\left(\nabla^{2} \mu_{c(s)}+\frac{\epsilon-\mu}{T} \nabla^{2} T\right),
\end{gathered}
$$

into the above diffusion equations we can integrate over energies by using the Sommerfeld approximation [see Eq. (3)]. We assume $S^{2} \ll \mathcal{L}_{0}$ and disregard an energy dependence of the spin-diffusion length $l_{\mathrm{sf}}$ (which is allowed when $2 P S_{\mathrm{sf}}$ $\ll\left(P^{\prime}-P\right) S$ in which $\left.S_{\mathrm{sf}}=-\left.e L_{0} T \partial_{\epsilon} \ln l_{\mathrm{sf}}(\epsilon)\right|_{\epsilon_{F}}\right)$ but keep the energy dependence of the spin polarization $P$ [recall Eq. (22)]. One then arrives at the thermoelectric spin-diffusion equations expressed in Eqs. (39)-(41). Among the spindiffusion equations Eqs. (39) and (40) are already well known. ${ }^{26}$ Equation (41) represents a spin-heat coupling for the electron spin diffusion in the presence of spin polarization of thermopower.
${ }^{1}$ F. Giazotto, T. T. Heikkilä, A. Luukanen, A. M. Savin, and J. P. Pekola, Rev. Mod. Phys. 78, 217 (2006).

${ }^{2}$ L. D. Hicks and M. S. Dresselhaus, Phys. Rev. B 47, 12727 (1993).

${ }^{3}$ B. C. Sales, Science 295, 1248 (2002).

${ }^{4}$ A. I. Boukai, Y. Bunimovich, J. Tahir-Kheli, J.-K. Yu, W. A. Goddard, and J. R. Heath, Nature (London) 451, 168 (2008).

${ }^{5}$ A. I. Hochbaum, R. Chen, R. D. Delgado, W. Liang, E. C. Garnett, M. Najarian, A. Majumdar, and P. Yang, Nature (London) 451, 163 (2008).

${ }^{6}$ R. Venkatasubramanian, E. Siivola, T. Colpitts, and B. O'Quinn, Nature (London) 413, 597 (2001).

${ }^{7}$ H. Ohta et al., Nature Mater. 6, 129 (2007).

${ }^{8}$ L. W. Molenkamp, T. Gravier, H. van Houten, O. J. A. Buijk, M. A. A. Mabesoone, and C. T. Foxon, Phys. Rev. Lett. 68, 3765 (1992).

${ }^{9}$ H. van Houten, L. W. Molenkamp, C. W. J. Beenakker, and C. T. Foxon, Semicond. Sci. Technol. 7, B215 (1992).

${ }^{10}$ M. Johnson and R. H. Silsbee, Phys. Rev. B 35, 4959 (1987).

${ }^{11}$ M. Johnson, J. Supercond. 16, 679 (2003).

${ }^{12}$ J.-E. Wegrowe, Phys. Rev. B 62, 1067 (2000).

${ }^{13}$ L. Gravier, S. Serrano-Guisan, F. Reuse, and J.-P. Ansermet, Phys. Rev. B 73, 024419 (2006).

${ }^{14}$ L. Gravier, S. Serrano-Guisan, F. Reuse, and J.-P. Ansermet, Phys. Rev. B 73, 052410 (2006).
${ }^{15}$ S. Serrano-Guisan, G. di Domenicantonio, M. Abid, J. P. Abid, M. Hillenkamp, L. Gravier, J.-P. Ansermet, and C. Félix, Nature Mater. 5, 730 (2006).

${ }^{16}$ O. Tsyplyatyev, O. Kashuba, and V. I. Fal'ko, Phys. Rev. B 74, 132403 (2006).

${ }^{17}$ M. Hatami, G. E. W. Bauer, Q. F. Zhang, and P. J. Kelly, Phys. Rev. Lett. 99, 066603 (2007).

${ }^{18}$ A. Fukushima, K. Yagami, A. A. Tulapurkar, Y. Suzuki, H. Kubota, A. Yamamoto, and S. Yuasa, Jpn. J. Appl. Phys., Part 2 44, L12 (2005).

${ }^{19}$ A. Fukushima, H. Kubota, A. Yamamoto, Y. Suzuki, and S. Yuasa, IEEE Trans. Magn. 41, 2571 (2005).

${ }^{20}$ L. Gravier, A. Fukushima, H. Kubota, A. Yamamoto, and S. Yuasa, J. Phys. D 39, 5267 (2006).

${ }^{21}$ H. Katayama-Yoshida, T. Fukushima, V. A. Dinh, and K. Sato, Jpn. J. Appl. Phys., Part 2 46, L777 (2007).

${ }^{22}$ Y. Dubi and M. Di Ventra, Phys. Rev. B 79, 081302(R) (2009).

${ }^{23}$ A. Brataas, Y. V. Nazarov, and G. E. W. Bauer, Phys. Rev. Lett. 84, 2481 (2000).

${ }^{24}$ A. Brataas, Y. V. Nazarov, and G. E. W. Bauer, Eur. Phys. J. B 22, 99 (2001).

${ }^{25}$ A. Brataas, G. E. W. Bauer, and P. J. Kelly, Phys. Rep. 427, 157 (2006).

${ }^{26}$ T. Valet and A. Fert, Phys. Rev. B 48, 7099 (1993).

${ }^{27}$ J. Bass and W. P. Pratt, Jr., J. Magn. Magn. Mater. 200, 274 
(1999).

${ }^{28}$ D. K. C. MacDonald, Thermoelectricity: An Introduction to the Principles (John Wiley \& Sons, New York, 1962).

${ }^{29}$ K. Xia, M. Zwierzycki, M. Talanana, P. J. Kelly, and G. E. W. Bauer, Phys. Rev. B 73, 064420 (2006).

${ }^{30}$ Q. F. Zhang, P. J. Kelly, M. Hatami, and G. E. W. Bauer (unpublished).

${ }^{31}$ K. M. Schep, J. B. A. N. van Hoof, P. J. Kelly, G. E. W. Bauer, and J. E. Inglesfield, Phys. Rev. B 56, 10805 (1997).

${ }^{32}$ N. W. Ashcroft and N. D. Mermin, Solid State Physics (HoltSaunders International Editions, Philadelphia, 1976).

${ }^{33}$ L. Colquitt, Jr., H. R. Fankhauser, and F. J. Blatt, Phys. Rev. B 4, $292(1971)$

${ }^{34}$ B. C. Gundrum, D. G. Cahill, and R. S. Averback, Phys. Rev. B 72, 245426 (2005)

${ }^{35}$ G. D. Guttman, E. Ben-Jacob, and D. J. Bergman, Phys. Rev. B 52, 5256 (1995).

${ }^{36}$ K. E. Nagaev, Phys. Rev. B 52, 4740 (1995).

${ }^{37}$ H. Pothier, S. Guéron, N. O. Birge, D. Esteve, and M. H. Devoret, Phys. Rev. Lett. 79, 3490 (1997).

${ }^{38}$ M. C. Cadeville and J. Roussel, J. Phys. F: Met. Phys. 1, 686 (1971).

${ }^{39}$ L. Piraux, A. Fert, P. A. Schroeder, R. Loloee, and P. Etienne, J. Magn. Magn. Mater. 110, L247 (1992).

${ }^{40}$ J. Shi, K. Pettit, E. Kita, S. S. P. Parkin, R. Nakatani, and M. B. Salamon, Phys. Rev. B 54, 15273 (1996).

${ }^{41}$ L. Gravier, A. Fábián, A. Rudolf, A. Cachin, J.-E. Wegrowe, and J.-P. Ansermet, J. Magn. Magn. Mater. 271, 153 (2004).

${ }^{42}$ M. Hatami, T. Heikkilä, and G. E. W. Bauer (unpublished).

${ }^{43}$ M. Zwierzycki, Y. Tserkovnyak, P. J. Kelly, A. Brataas, and G. E. W. Bauer, Phys. Rev. B 71, 064420 (2005).

${ }^{44}$ S. Urazhdin, R. Loloee, and W. P. Pratt, Jr., Phys. Rev. B 71, 100401(R) (2005).

${ }^{45}$ A. A. Kovalev, G. E. W. Bauer, and A. Brataas, Phys. Rev. B 73, 054407 (2006).

${ }^{46}$ M. Zwierzycki et al., Phys. Status Solidi B 245, 623 (2008).
${ }^{47}$ I. Turek, V. Drchal, J. Kudrnovský, M. Šob, and P. Weinberger, Electronic Structure of Disordered Alloys, Surfaces and Interfaces (Kluwer, Boston, London, Dordrecht, 1997).

${ }^{48}$ O. K. Andersen and O. Jepsen, Phys. Rev. Lett. 53, 2571 (1984).

${ }^{49}$ O. K. Andersen, Z. Pawlowska, and O. Jepsen, Phys. Rev. B 34, 5253 (1986).

${ }^{50}$ T. Ando, Phys. Rev. B 44, 8017 (1991).

${ }^{51}$ K. Xia, P. J. Kelly, G. E. W. Bauer, I. Turek, J. Kudrnovský, and V. Drchal, Phys. Rev. B 63, 064407 (2001).

${ }^{52}$ P. Soven, Phys. Rev. 156, 809 (1967).

${ }^{53}$ K. Xia, P. J. Kelly, G. E. W. Bauer, A. Brataas, and I. Turek, Phys. Rev. B 65, 220401(R) (2002).

${ }^{54}$ R. J. Gripshover, J. B. VanZytveld, and J. Bass, Phys. Rev. 163, 598 (1967).

${ }^{55}$ J. Bass, Thermoelectricity, AccessScience (McGraw-Hill, New York, 2000).

${ }^{56}$ Y. G. Gurevich and G. N. Logvinov, Semicond. Sci. Technol. 20, R57 (2005).

${ }^{57}$ V. R. F. J. Blatt, D. J. Flood, and P. A. Schroeder, Phys. Rev. Lett. 18, 395 (1967).

${ }^{58}$ F. J. Blatt, Thermoelectric Power of Metals (Plenum, New York, 1976).

${ }^{59}$ M. Jonson and G. D. Mahan, Phys. Rev. B 21, 4223 (1980).

${ }^{60}$ H. Kontani, Phys. Rev. B 67, 014408 (2003).

${ }^{61}$ A. A. Kovalev, A. Brataas, and G. E. W. Bauer, Phys. Rev. B 66, 224424 (2002).

${ }^{62}$ F. J. Albert, J. A. Katine, R. A. Buhrman, and D. C. Ralph, Appl. Phys. Lett. 77, 3809 (2000).

${ }^{63}$ R. H. Koch, J. A. Katine, and J. Z. Sun, Phys. Rev. Lett. 92, 088302 (2004)

${ }^{64}$ R. J. Soulen Jr. et al., Science 282, 85 (1998).

${ }^{65}$ K. Uchida, S. Takahashi, K. Harii, J. Ieda, W. Koshibae, K. Ando, S. Maekawa, and E. Saitoh, Nature (London) 455, 778 (2008).

${ }^{66}$ R. H. M. Groeneveld, R. Sprik, and A. Lagendijk, Phys. Rev. B 51, 11433 (1995). 